\title{
Current achievements and future perspectives for projection-based particle methods with applications in ocean engineering
}

\author{
Hitoshi Gotoh $^{1} \cdot$ Abbas Khayyer $^{1}$
}

Received: 30 November 2015 / Accepted: 17 March 2016 / Published online: 14 April 2016

(C) Springer International Publishing Switzerland 2016

\begin{abstract}
The paper aims at providing a comprehensive and up-to-date review on the latest achievements made in the context of particle methods, in particular the projectionbased ones, with applications in ocean engineering. The latest achievements corresponding to stability, accuracy, energy conservation and boundary condition enhancements as well as advancements related to improved simulations of multiphase flows, surface tension and fluid-structure interactions are reviewed. The future perspectives for enhancement of applicability and reliability of these methods for ocean engineering applications are also highlighted.
\end{abstract}

Keywords Particle methods - Projection method . Incompressible SPH - Moving particle semi-implicit . Stability · Accuracy $\cdot$ Energy conservation · Boundary condition · Multiphase flow · Fluid-structure interaction . Scouring $\cdot$ Suspended sediment

\section{Introduction}

Due to their mesh-free, Lagrangian nature, particle methods have been proven to provide a substantial potential for simulation of free-surface fluid flows and their interactions with the environment that are often encountered in ocean engineering. During the past two decades a vast number of

Hitoshi Gotoh

gotoh@particle.kuciv.kyoto-u.ac.jp

1 Department of Civil and Earth Resources Engineering, Kyoto University, Katsura Campus, Nishikyo-ku, Kyoto 615-8540, Japan researches have been conducted on development and application of particle methods, including both SPH (smoothed particle hydrodynamics; Gingold and Monaghan 1977) and MPS (moving particle semi-implicit; Koshizuka and Oka 1996) methods, for different fields of engineering, including ocean engineering. These researches were mainly focused on enhancements of stability/accuracy as well as extension of applications, including ocean engineering related ones.

The existing applications of particle methods in ocean engineering include wave breaking (e.g. Gotoh and Sakai 1999; Khayyer and Gotoh 2008; Farahani and Dalrymple 2014), wave overtopping (e.g. Gotoh et al. 2005; Shao et al. 2006), wave run-up (e.g. Shadloo et al. 2015), wave impact (e.g. Khayyer and Gotoh 2009a; Lee et al. 2011; Altomare et al. 2015), wave-induced nearshore circulation system (Farahani et al. 2014), violent sloshing (e.g. Delorme et al. 2009; Gotoh et al. 2014), oil spilling (e.g. Violeau et al. 2007), green water on ships (e.g. Shibata and Koshizuka 2007; Le Touzé et al. 2010), sediment transport (e.g. Gotoh and Sakai 2006), landslide-generated waves (e.g. Panizzo and Dalrymple 2004; Fu and Jin 2015) and fluid-structure interactions (e.g. Rafiee and Thiagarajan 2009; Shibata et al. 2012; Hwang et al. 2014; Colagrossi et al. 2015; Wei et al. 2015).

In general, particle methods applied for free-surface fluid flows can be categorized into two groups of weakly compressible and incompressible ones. The weakly compressible particle methods such as Weakly Compressible SPH (WCSPH; e.g. Colagrossi and Landrini (2003); Dalrymple and Rogers 2006) or Weakly Compressible MPS (WCMPS; e.g. Shakibaeinia and Jin 2012; Tayebi and Jin 2015) methods solve an appropriate equation of state in a fully explicit form. The incompressible particle methods such as MPS or incompressible SPH (ISPH; e.g. Shao and Lo 2003) methods solve a Poisson pressure equation (PPE) through a HelmholtzHodge decomposition and application of Chorin's projection 
method (Chorin 1968). Hence, they can be referred to as projection-based particle methods. The concept of Chorin's projection is illustrated for both ISPH and MPS methods by Khayyer and Gotoh (2009b) and Khayyer and Gotoh (2011).

Several studies have compared the performance of ISPH with respect to WCSPH (e.g. Lee et al. 2008; Hughes and Graham 2010; Khayyer and Gotoh 2010a; Shadloo et al. 2012; Zheng et al. 2014a). In general, projection-based particle methods are expected to provide higher accuracy in terms of pressure calculation and volume conservation (Gotoh et al. 2013). However, from computational point of view, solving a PPE may bring about distinct challenges, especially for parallelized and/or GPU-based computations (e.g. Hori et al. 2011).

This paper aims at reviewing the latest achievements made in the field of particle methods, especially the projectionbased ones with applications in ocean engineering. The ongoing researches and future perspectives will be also discussed. The latest achievements correspond to enhancements of stability, accuracy, energy conservation, boundary conditions and improved simulations of multiphase flows, surface tension, fluid-structure interactions, etc. Despite the advancements made, several key aspects still remain to be not comprehensively resolved. Examples of such remaining unresolved issues include stability, convergence, adaptivity, boundary conditions and consistency/conservation. The first four issues are considered as SPH grand challenges by the SPHERIC (SPH European Research Interest Community).

\section{Latest achievements}

In general, both weakly compressible and incompressible particle methods for free-surface fluid flows provide solutions, on the basis of particle-based discretizations, to the continuity and Navier-Stokes equations that are expressed as follows, in a continuous framework:

$$
\begin{aligned}
& \frac{1}{\rho} \frac{\mathrm{D} \rho}{\mathrm{D} t}+\nabla \cdot \boldsymbol{u}=0 \\
& \frac{\mathrm{D} \boldsymbol{u}}{\mathrm{D} t}=-\frac{1}{\rho} \nabla p+\boldsymbol{g}+v \nabla^{2} \boldsymbol{u},
\end{aligned}
$$

where $\boldsymbol{u}$ denotes particle velocity vector; $t$ stands for time; $\rho$ represents fluid density; $p$ symbolizes particle pressure; $\boldsymbol{g}$ signifies gravitational acceleration vector and $v$ represents laminar kinematic viscosity. It should be noted that Eq. 1 is written in the form of a compressible flow. In projectionbased particle methods, incompressibility is enforced by setting $\mathrm{D} \rho / \mathrm{D} t$ equal to zero at each particle at each calculation time step through application of Helmholtz-Hodge decomposition and a prediction-correction process. The latest advancements corresponding to particle methods, and in particular projection-based ones, in solving the abovementioned governing equations (together with other related governing equations) are discussed in this section.

\subsection{Stability enhancement}

The stability issue is of crucial importance for proper and reliable application of particle methods to engineering problems including those encountered in ocean engineering. In general, the numerical instabilities associated with particle methods can be categorized into two major categories of rank deficiency and stress state instabilities. The rank deficiency instability is related to spurious singular or zero-energy modes occurring when the field variables and their derivatives are calculated at the same calculation points (Beissel and Belytschko 1996). This particular instability is not limited to particle methods and it can be found in grid-based methods including finite element and finite difference methods (Vignjevic 2004). As the name indicates, stress state instabilities, including so-called compressive and tensile instabilities, depend on the state of stress and growth of perturbations with kernel-based approximations of inter-particle interactions. The compressive instability occurs in the presence of repulsive inter-particle forces when inter-particle interaction strength decreases as the particles approach (Swegle et al. 1994; Johnson et al. 1996). On the other hand, tensile instability occurs in the presence of attractive inter-particle forces when inter-particle interaction strength increases as the particles approach (Swegle et al. 1994; Swegle 2000; Khayyer and Gotoh 2011).

By performing a one-dimensional von Neumann stability analysis for the SPH method, Swegle et al. (1995) found a criterion for an unstable growth of perturbations based on the sign of the stress and kernel's second derivative. A similar criterion was identified for stability of MPS by Khayyer and Gotoh (2011). Several studies (e.g. Balsara 1995; Morris 1996; Robinson 2009; Dehnen and Aly 2012) highlighted the significance of the Fourier transform of the kernel function in stability properties of SPH. However, none of the above mentioned papers provided an explicit criterion for the maximum allowable time step.

Morris et al. (1997) proposed a criterion for maximum allowable time step in WCSPH context. Through performing a rigorous theoretical stability analysis for unbounded flows, Violeau and Leroy (2014) derived an analytical formula for the stability condition and thus the maximum allowable time step for WCSPH. They later extended their rigorous work to ISPH (Violeau and Leroy 2015). The maximum CFL number for ISPH at large Reynolds numbers was found to be twice smaller than that of WCSPH and thus, resulting in an optimal time step size of only five times larger for ISPH.

There have been a wide range of efforts to minimize the possibility of occurrence of instabilities in particle meth- 
ods, including those targeting tensile instability. However, as highlighted by Belytschko and Xiao (2002), perfect elimination of tensile instability appears to be unachievable as long as an Eulerian kernel is used with a purely Lagrangian description of motion. Thus, such instability tends to arise in both weakly compressible particle methods as well as projectionbased ones. Belytschko and Xiao (2002) showed that tensile instability can be eliminated when the kernel is a function of material coordinates (i.e. a Lagrangian kernel). The problem related to the Lagrangian kernels is that they may not tolerate large deformations as in case of fluid flows (Belytschko and Xiao 2002; Rabczuk et al. 2004), particularly the violent ones. The efforts corresponding to minimization of probability of tensile instability occurrence can be categorized into the following distinct groups:

\section{(i) Artificial repulsive forces}

To resolve the problem of tensile instability in SPH, Monaghan (2000) and Gray et al. (2001) proposed artificial repulsive forces proportional to the fluid pressure and the stress tensor, respectively. In particular, in fluidstructure interaction (FSI) simulations, such kind of treatment has been repeatedly used to ensure the stability of calculations (e.g. Antoci et al. 2007; Rafiee and Thiagarajan 2009; Kondo et al. 2010). As shown by Tsuruta et al. (2013), application of artificial repulsive forces may adversely affect the reproduced physics of simulations, in particular due to possible generation of excessive repulsive forces that are more adequate for numerical stabilization. Tsuruta et al. (2013) presented a so-called dynamic stabilization (DS) scheme which is aimed to produce exactly adequate repulsive forces to ensure the numerical stability. The applicability and effectiveness of this scheme has to be further examined for a wider range of free-surface, internal and multiphase flows.

(ii) Corrective functions for enhancements of kernel estimates

Dilts (1999) showed that accurate estimation of derivatives is a key point in removal of tensile instability. This is mainly due to the fact that tensile instability is triggered when unphysical perturbations in particle motions exist. Khayyer and Gotoh (2011) proposed a gradient correction (GC) for MPS method to minimize the unphysical perturbations in particle motions and achieved improved stability performance. Similar approaches have been introduced in the context of SPH method. For instance, Chen et al. (1999) proposed a corrective $\mathrm{SPH}(\mathrm{CSPH})$ to improve the stability of SPH. In projection-based particle methods, corrective or error minimizing schemes can be introduced in the source term of Poisson pressure equation (PPE) to minimize the projection-related errors to achieve enhanced pres- sure field and uniform particle distributions throughout the simulation that minimizes the perturbations in particle motions. For instance, Khayyer and Gotoh (2011) introduced so-called error compensating source (ECS) terms of PPE with dynamic coefficients as functions of instantaneous flow features. The ECS scheme could be considered as an enhanced and updated version of the scheme proposed by Kondo and Koshizuka (2011).

(iii) Conservative smoothing

Based on the von Neumann-Richtmyer discrete representation of conservation of volume, Guenther et al. (1994) presented a conservative smoothing formalism for SPH. They showed that a proper conservative smoothing produces significantly more stable and accurate solutions compared to commonly used artificial viscosity. The effectiveness of conservative smoothing in minimization of occurrence probability of tensile instability is proved in the studies by Hicks and Liebrock (2004) and Xu et al. (2008). To the best knowledge of authors, the conservative smoothing technique has not been applied yet within the framework of projectionbased particle methods. However, its applicability can be tested for applications that are prone to numerical instability, for instance, multiphase flows with high density ratios.

(iv) Stress-points

Dyka et al. (1997) proposed an alternative approach to tackle the problem of tensile instability in particle methods. This approach was founded on introduction of a set of additional particles in between the original particles to serve as additional quadrature points. Randles and Libersky (2000) later extended this method to higher dimensions. Belytschko et al. (2000) showed that the stress point technique stabilizes SPH by removing the instability that arises due to rank deficiency, while the stress state related tensile instability can be avoided only by using a Lagrangian kernel.

(v) Lagrangian kernels

As previously stated, although a careful implementation of stress points removes the zero-energy modes, it does not eliminate the tensile instability (Belytschko et al. 2000). Belytschko and Xiao (2002) highlighted the fact that tensile instability occurs when an Eulerian kernel is used with a Lagrangian description of motion. They showed that this instability is eliminated when the kernel is a function of material coordinates (i.e. a Lagrangian kernel). It was also found that the best approach to stabilize particle-based methods is to use Lagrangian kernels with stress points. However, apart from the increased complexity of mathematical formulations, in case of application of stress points, the stability and convergence would depend on the distribution of particles in the domain, where a poor convergence rate 
would be obtained for irregular particle distributions (Fries and Belytschko 2008). Furthermore, Lagrangian kernels do not appear to be proper for problems involving large deformations, as in case of free-surface fluid flows.

(vi) Total Lagrangian formalism

Vignjevic et al. (2006) showed that the tensile instability can be resolved through a total Lagrangian description of continuum. They also introduced consistency corrections into the total Lagrangian SPH formalism to enhance the accuracy of their SPH solid mechanics simulations.

It should be noted here that to the best knowledge of authors, stress points, Lagrangian kernels and total Lagrangian formalism have not been tested yet in the context of projection-based particle methods. However, all of the mentioned techniques appear to be applicable within this context as well, for instance, in FSI simulations by a coupled fully Lagrangian solver comprising of a projection-based fluid model and an elastic structure model. These techniques tend to stabilize the structure model, and thus the overall FSI solver, by removing the spurious zero-energy modes and/or minimizing incidence of tensile instability.

In addition to the abovementioned approaches, a distinct category of schemes, proven to be effective for both stability and accuracy enhancement of particle methods, corresponds to the particle regularization schemes. A concise review of this class of schemes is presented in Sect. 2.2.2.

\subsection{Accuracy enhancement}

One of the main shortcomings of particle methods, including projection-based ones, corresponds to presence of unphysical pressure oscillations (e.g. Gotoh et al. 2005, 2013; Khayyer and Gotoh 2009a, b). This shortcoming could have limited the application of particle methods to ocean engineering. However, there have been substantial efforts and progresses corresponding to this distinct shortcoming (e.g. Ataie-Ashtiani et al. 2008; Khayyer et al. 2009; Koshizuka 2011; Gotoh et al. 2014). These efforts could result in reliable particle methods that provide acceptable solutions to the considered governing equations.

In the context of weakly compressible $\mathrm{SPH}$, the so-called delta-SPH (Antuono et al. 2012) as well as Riemann SPH (Inutsuka 1994, 2002; Monaghan 1997; Gao et al. 2012; Rafiee et al. 2012) schemes have been proposed to enhance the accuracy, especially in terms of reproduced pressure field. Several rigorous studies also investigate the accuracy of SPH and highlight the importance of higher order interpolation schemes to improve the method's performance (e.g. Le Touzé et al. 2013). Corrected SPH methods with corrective terms to restore the completeness or consistency (Colagrossi et al.
2011) of formulations (e.g. Randles and Libersky 1996; Chen et al. 1999; Oger et al. 2007; Schwaiger 2008; Fatehi and Manzari 2011a; Jiang et al. 2012) as well as momentum conservation (e.g. Bonet and Lok 1999; Hopkins 2015) have also been developed and applied to ocean engineering problems (e.g. Sun et al. 2010; Xie et al. 2012).

As for projection-based particle methods, refined differential operator models have been proposed for discretization of source term and Laplacian of PPE as well as corrective terms to restore consistency of approximations (e.g. Khayyer and Gotoh 2011; Ikari et al. 2015a) and momentum conservation (e.g. Khayyer et al. 2008; Khayyer and Gotoh 2008). Khayyer and Gotoh (2009a) proposed a Higher-order Source term of PPE abbreviated as HS scheme. Later in 2010, a Higher-order Laplacian (HL) model was proposed (Khayyer and Gotoh 2010b) to further enhance the pressure field calculations. The HL scheme was extended to three dimensions (Khayyer and Gotoh 2012) and its enhancing performance with respect to the standard MPS Laplacian was demonstrated by a number of benchmark tests including ocean engineering related ones. To enhance volume conservation and projection-related errors, the ECS (error compensating source of PPE) was proposed for both MPS (Khayyer and Gotoh 2011) and ISPH (Gotoh et al. 2014) methods. The ISPH version of HL was also shown to provide improved results with respect to the commonly applied hybrid SPH-finite difference Laplacian model of SPH (Shao and Lo 2003) in simulation of violent sloshing flows (Gotoh et al. 2014).

Recently, Zheng et al. (2014b) proposed a new ISPH based on Rankine source solution that transforms the PPE into a form that does not require any direct approximations for function derivatives. The advantage of the so-called ISPHR (ISPH with Rankine source solution) mainly corresponds to absence of the need to approximate second-order derivatives in the PPE. The enhanced performance of ISPH-R with respect to standard ISPH was shown through a number of benchmark tests including those related to water waves and violent sloshing flows. Ngo-Cong et al. (2015) proposed an improved ISPH method through solving the PPE on a set of so-called moving integrated radial basis function networks.

Among other impressive works corresponding to accuracy enhancement in the context of projection-based particle methods, we can mention the multiphase projection formulation of $\mathrm{Hu}$ and Adams (2007), in which both the zerodensity-variation and velocity-divergence-free constraints of the incompressibility condition were enforced through the resolution of two PPEs and via application of a fractional time-step integration algorithm.

Two different classes of schemes corresponding to accuracy and stability enhancements of projection-based particle methods, namely refined schemes and particle regularization schemes are briefly reviewed in Sects. 2.2.1 and 2.2.2, respectively. 


\subsubsection{Refined schemes for accuracy and stability enhancements}

\subsubsection{HS and HL schemes}

In general, in projection-based particle methods the PPE is formulated as follows (Gotoh 2009; Khayyer and Gotoh 2011):

$$
\begin{aligned}
& \left\langle\nabla^{2} p_{k+1}\right\rangle_{i}=\frac{1}{\Delta t}\left(\frac{\mathrm{D} \rho}{\mathrm{D} t}\right)_{i}^{*} ; \\
& \rho=m \sum_{i \neq j} w\left(\left|\boldsymbol{r}_{i j}\right|\right)=m \sum_{i \neq j} w_{i j} ; \quad \boldsymbol{r}_{i j}=\boldsymbol{r}_{j}-\boldsymbol{r}_{i},
\end{aligned}
$$

where $m$ denotes particle mass, $\boldsymbol{r}$ presents the particle position vector, $w$ presents kernel function, $k$ signifies the calculation step number and $\Delta t$ symbolizes the calculation time step. In Eq. 3, $i$ and $j$ represent a target particle $i$ and a typical neighboring particle $j$. The superscript ${ }^{*}$ denotes the pseudo-time step $k+1 / 2$, corresponding to the end of prediction step. Considering the concept of particle number density, $n$, in MPS, the PPE is written as

$$
\left\langle\nabla^{2} p_{k+1}\right\rangle_{i}=\frac{\rho}{n_{0} \Delta t}\left(\frac{\mathrm{D} n}{\mathrm{D} t}\right)_{i}^{*} ; \quad n=\sum_{i \neq j} w\left(\left|\boldsymbol{r}_{i j}\right|\right)=\sum_{i \neq j} w_{i j},
$$

where $n_{0}$ represents the reference particle number density, $n$. Discretization of the source term of PPE (right-hand side of Eq. 4) and the Laplacian of pressure (left-hand side of Eq. 4) by HS (higher order source; Khayyer and Gotoh 2009a) and HL (higher order Laplacian; Khayyer and Gotoh 2010b, 2012) schemes are conducted as follows:

$$
\begin{aligned}
& \left(\frac{\mathrm{D} n}{\mathrm{D} t}\right)_{i}^{*}=\sum_{j \neq i}\left(\frac{\partial w_{i j}}{\partial r_{i j}}\right) \frac{\boldsymbol{r}_{i j} \cdot \boldsymbol{u}_{i j}^{*}}{\left|\boldsymbol{r}_{i j}\right|} \\
& \left\langle\nabla^{2} p_{k+1}\right\rangle_{i} \\
& =\frac{1}{n_{0}} \sum_{j \neq i}\left\{\frac{\partial p_{i j}}{\partial r_{i j}} \frac{\partial w_{i j}}{\partial r_{i j}}+p_{i j}\left(\frac{\partial^{2} w_{i j}}{\partial r_{i j}^{2}}+\frac{D_{s}-1}{r_{i j}} \frac{\partial w_{i j}}{\partial r_{i j}}\right)\right\},
\end{aligned}
$$

where $u$ and $v$ denote horizontal and vertical components of velocity vector $\boldsymbol{u}, p_{i j}=p_{j}-p_{i} ; r_{i j}=r_{j}-r_{i} ; r=$ $|\boldsymbol{r}| ; u_{i j}=u_{j}-u_{i}$ and $v_{i j}=v_{j}-v_{i}$. The variable $D_{s}$ in Eq. 6 corresponds to the number of space dimensions. Recently, Ikari et al. (2015b) presented a corrected HL (CHL) scheme by carefully taking the divergence of a corrected gradient model. The enhanced performance of CHL with respect to HL could be verified, especially for calculation cases with irregular initial arrangements.

\subsubsection{ECS scheme}

As previously stated and as it has been explained in details by Khayyer and Gotoh (2011), discretization of the source term of PPE even by accurate differential operator models does not guarantee a divergence-free velocity field corresponding to an incompressible fluid flow. The ECS (error compensating source) scheme proposed by Khayyer and Gotoh (2011) is written in the following form:

$$
\begin{gathered}
\left\langle\nabla^{2} p_{k+1}\right\rangle_{i}=\frac{\rho}{n_{0} \Delta t}\left(\frac{D n}{D t}\right)_{i}^{*}+\Lambda_{\mathrm{ECS}} \\
\Lambda_{\mathrm{ECS}}=\frac{\rho}{\Delta t}\left\{\frac{\alpha}{n_{0}}\left(\frac{D n}{D t}\right)_{i}^{k}+\frac{\beta}{\Delta t} \frac{n_{i}^{k}-n_{0}}{n_{0}}\right\} ; \\
\alpha=\left|\frac{n_{i}^{k}-n_{0}}{n_{0}}\right| ; \beta=\left|\frac{\Delta t}{n_{0}}\left(\frac{D n}{D t}\right)_{i}^{k}\right|
\end{gathered}
$$

Accordingly, the source term of PPE will be comprised of a high-order main term (HS scheme) and two error mitigating terms multiplied by dynamic coefficients $(\alpha, \beta)$ as functions of instantaneous flow field. In Eq. 7, the first error mitigating term (which is multiplied by coefficient $\alpha$ ) corresponds to the instantaneous time variation of particle density at time step $k$. The second term (which is multiplied by coefficient $\beta$ ) reflects the deviation of particle density at time step $k$ from the theoretical constant one $\left(\rho_{0}\right)$. In other words, the firstand second-error mitigating terms correspond to the instantaneous and accumulative density deviations, respectively. The dynamic coefficients adjust the intensities of these two error mitigating terms depending on the instantaneous state of flow field. Similar ECS scheme has been formulated and validated for the ISPH (Gotoh et al. 2014).

\subsubsection{GC and DS schemes}

A proper Taylor-series consistent pressure gradient model with gradient correction (GC; Khayyer and Gotoh 2011) and dynamic stabilization (DS; Tsuruta et al. 2013) schemes is expressed as follows:

$\left\langle\frac{\nabla p}{\rho}\right\rangle_{i}=\frac{D_{s}}{\rho n_{0}} \sum_{j \neq i} \frac{p_{j}-p_{i}}{\left|\boldsymbol{r}_{i j}\right|^{2}} \boldsymbol{C}_{i} \boldsymbol{r}_{i j} w_{i j}+\Lambda_{\mathrm{DS}}$,

where the gradient correction (GC) matrix, $\boldsymbol{C}_{i}$, is expressed as follows:

$\boldsymbol{C}_{i}=\frac{1}{D_{s}}\left(V_{i} \sum_{j \neq i} \frac{\boldsymbol{r}_{i j} \otimes \boldsymbol{r}_{i j}}{\left|\boldsymbol{r}_{i j}\right|^{2}} w_{i j}\right)^{-1} ; \quad V_{i}=\frac{1}{\sum_{j \neq i} w_{i j}}$

In Eq. 8, the DS scheme (Tsuruta et al. 2013) is formulated as follows: 
(a)
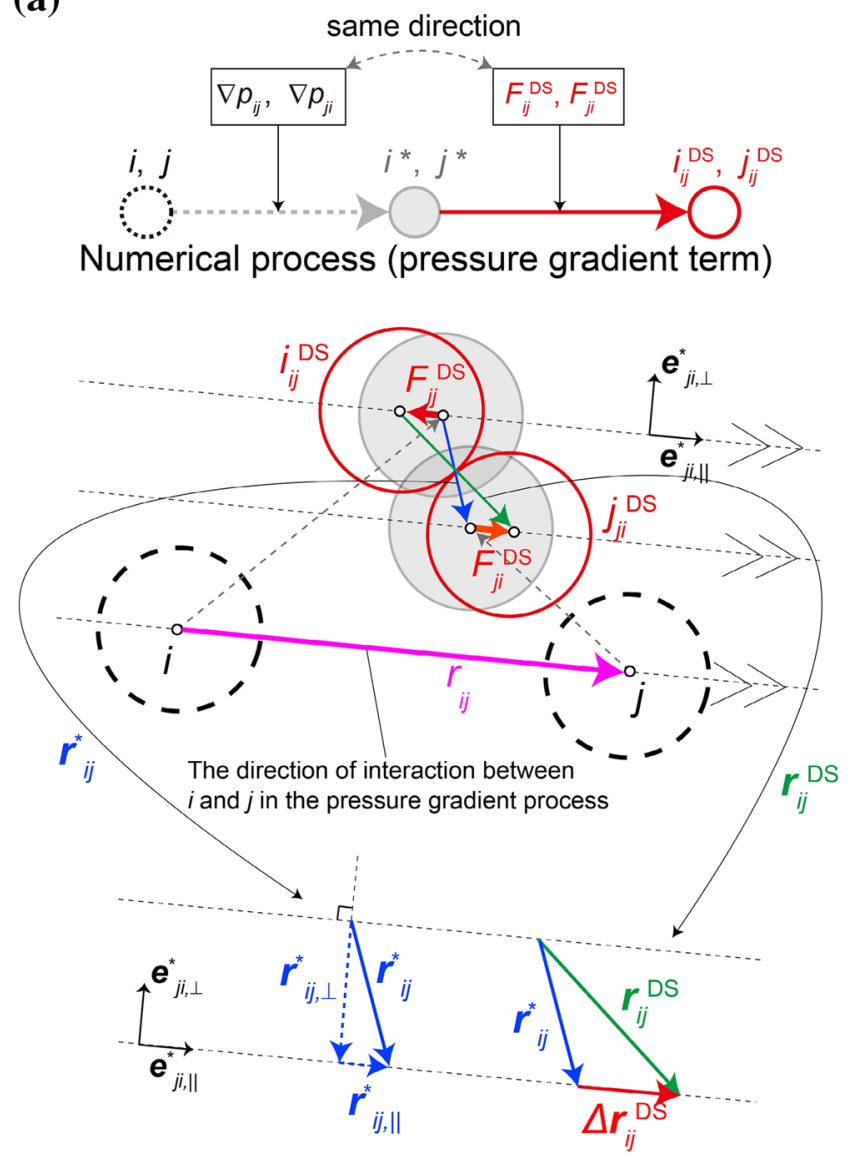

(b)

\section{CMPS-HS}
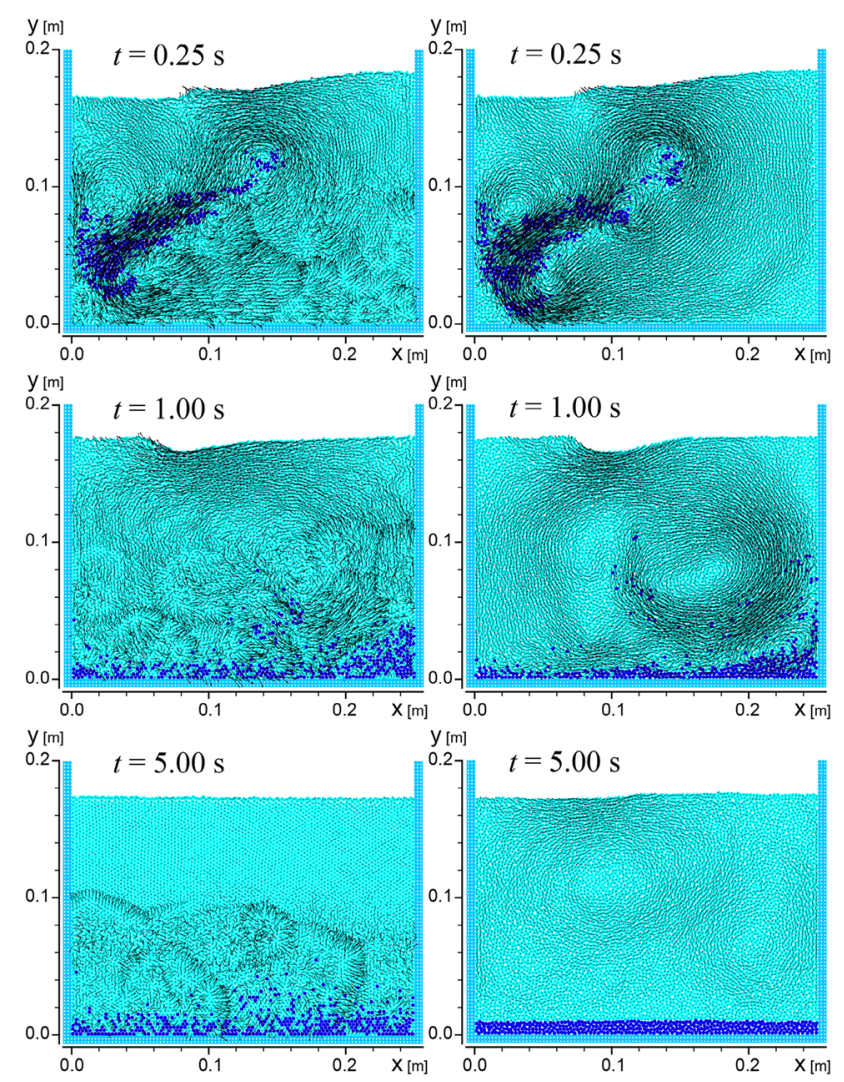

Fig. 1 a A graphical representation of the concept of DS (dynamic stabilization) scheme, b effectiveness of DS scheme in proper modeling of settlement of heavy particles in water (Tsuruta et al. 2013)

$$
\begin{aligned}
& \Lambda_{\mathrm{DS}}=V_{i} \sum_{j \neq i} \boldsymbol{F}_{i j}^{\mathrm{DS}} w_{i j} \quad ; \\
& \boldsymbol{F}_{i j}^{\mathrm{DS}}= \begin{cases}0 & \left|\boldsymbol{r}_{i j}^{*}\right| \geq d_{i j} \\
-\rho_{i} \Pi_{i j} \frac{\boldsymbol{r}_{i j}}{\left|\boldsymbol{r}_{i j}\right|} & \left|\boldsymbol{r}_{i j}^{*}\right|<d_{i j}\end{cases} \\
& d_{i j}=\alpha_{\mathrm{DS}} \frac{d_{i}+d_{j}}{2} ; \quad \alpha_{\mathrm{DS}}=1-\alpha_{d t} \\
& \Pi_{i j}=\frac{\rho_{j}}{(\Delta t)^{2}\left(\rho_{i}+\rho_{j}\right)}\left(\sqrt{d_{i j}^{2}-\left|\boldsymbol{r}_{i j \perp}^{*}\right|^{2}}-\left|\boldsymbol{r}_{i j \|}^{*}\right|\right),
\end{aligned}
$$

where $\boldsymbol{F}_{i j}^{\mathrm{DS}}$ is the stabilizing force for target particle $i$ from its neighboring particle $j ; \Pi_{i j}$ is the parameter to adjust the magnitude of $\boldsymbol{F}_{i j}^{\mathrm{DS}} ; \alpha_{\mathrm{DS}}$ is a constant for adjusting active range of $\boldsymbol{F}_{i j}^{\mathrm{DS}} ; \alpha_{d t}$ is the ratio of the time step to Courant number; $d$ represents the particle diameter; $\boldsymbol{r}_{i j \|}^{*}$ is the parallel vector of $\boldsymbol{r}_{i j}^{*}$ and $\boldsymbol{r}_{i j \perp}^{*}$ is the normal vector of $\boldsymbol{r}_{i j}^{*}$ with $\boldsymbol{r}_{i j}^{*}=\boldsymbol{r}_{i j \|}^{*}+$ $r_{i j \perp}^{*}$. Figure 1 shows a graphical representation of the con- cept of DS scheme as well as its effectiveness in providing proper settlement of heavy particles in water. The so-called CMPS-HS (Khayyer and Gotoh 2009a) has not been able to reproduce this settlement due to excessive repulsive forces corresponding to a repulsive pressure gradient model. Details of this numerical test are provided in the paper by Tsuruta et al. (2013). The corresponding simulations were conducted by a total number of 7000 particles with diameter of $2.5 \mathrm{~mm}$. Densities of the light and heavy particles were set as 1000 and $2650 \mathrm{~kg} / \mathrm{m}^{3}$, respectively.

Without application of DS scheme, the stability of simulations performed by a Taylor-series consistent pressure gradient model is generally not guaranteed. Thus, purely repulsive (and conditionally Taylor-series consistent; Khayyer and Gotoh 2013) pressure gradient models were suggested to be used. For instance, a commonly applied MPS gradient model that results in purely repulsive pressure interacting forces is (Koshizuka et al. 1998)

$$
\left\langle\frac{\nabla p}{\rho}\right\rangle_{i}=\frac{D_{s}}{\rho n_{0}} \sum_{j \neq i} \frac{p_{j}-\hat{p}_{i}}{\left|\boldsymbol{r}_{i j}\right|^{2}} \boldsymbol{r}_{i j} w_{i j}
$$


$\hat{p}_{i}=\min _{j \in J}\left(p_{i}, p_{j}\right) ; \quad J=\left\{j: w_{i j} \neq 0\right\}$

In the context of ISPH, the specific formulations of HS, HL and ECS are given by Gotoh et al. (2014). Here, a considered corrected Taylor-series consistent pressure gradient model with DS scheme is formulated.

$$
\left\langle\frac{\nabla p}{\rho}\right\rangle_{i}=\sum_{j \neq i} \frac{m_{j}}{\rho_{i} \rho_{j}}\left(p_{j}-p_{i}\right) \boldsymbol{C}_{i} \nabla_{i} w_{i j}+\Lambda_{\mathrm{DS}},
$$

where $\nabla_{i} w_{i j}$ denotes the gradient of weight function $w_{i j}$ calculated at the target particle $i$. In Eq. 12, the gradient correction matrix is written as follows:

$\boldsymbol{C}_{i}=\left(V_{i} \sum_{j \neq i} \nabla w_{i j} \otimes \boldsymbol{r}_{i j}\right)^{-1}$

A symmetric repulsive pressure gradient model frequently applied in ISPH simulations because of its superior stability features (e.g. Shao and Lo 2003; Khayyer et al. 2008; Lee et al. 2008) is expressed as follows:

$\left\langle\frac{\nabla p}{\rho}\right\rangle_{i}=\sum_{j \neq i} m_{j}\left(\frac{p_{j}}{\rho_{j}^{2}}+\frac{p_{i}}{\rho_{i}^{2}}\right) \nabla w_{i j}$

As will be discussed in Sect. 2.3, repulsive pressure gradient models including Eqs. 11 and 14 result in a remarkably inferior energy conservation. Hence, they appear not to be thoroughly reliable, especially for applications where energy conservation properties become important.

\subsubsection{Particle regularization schemes}

A distinct category of methods developed for enhancement of both accuracy and stability for both explicit and semiimplicit projection-based particle methods correspond to particle regularization schemes that tend to regularize the anisotropic distributions of particles prone to be formed due to Lagrangian characteristics of particle methods. The most well-known and the simplest approach is the so-called XSPH scheme (Monaghan 1992) which helps the particles to move with a velocity close to that of their neighboring particles. Thus, the XSPH scheme improves the smoothness of velocity field. However, it is based on an arbitrarily tuned velocity smoothing, may lead to numerical dispersions (Fatehi and Manzari 2011b) and inaccurate results in case of sharp velocity gradients (Shahriari et al. 2013). Monaghan (2005) highlighted the fact that the XSPH scheme does not conserve energy and proposed an implicit XSPH to resolve this issue.

A relatively new particle regularization technique is the particle shifting scheme of Xu et al. (2009) which slightly shifts the particles to prevent anisotropic particle structures. A generalized version of this scheme has been proposed by Lind et al. (2012), allowing extended applications to freesurface flows. The particle shifting scheme is founded on Fick's diffusion law and relies on a Taylor expansion for evaluation of particle quantities in new positions. Despite its simplicity and effectiveness, the particle shifting scheme may violate the overall conservation properties (Lind et al. 2012) including conservations of momentum and energy. On the contrary, the DS scheme, which can also be considered as a particle regularization scheme, provides radial and anti-symmetric inter-particle forces and thus, at least, this scheme preserves both linear and angular momentum exactly. A detailed comparative study in between DS and particle shifting is currently being conducted by the authors.

In the context of weakly compressible SPH, Adami et al. (2013) proposed a particle velocity correction together with a consistent additional term in the momentum equation to take into account the required modification of the advection velocity. The scheme was proven to be effective in enhancing the accuracy and stability of internal flows. However, extensions to free-surface flows does not appear to be straightforward. Recently, Oger et al. (2015) proposed a specific transport velocity within an ALE formalism where the method is shown to be robust and accurate for both internal and free-surface flows. This scheme appears to be applicable within the context of projection-based particle methods to resolve the issue with anisotropic particle distributions prone to be formed due to purely Lagrangian descriptions.

\subsection{Energy conservation improvement}

In the context of weakly compressible SPH, Fang et al. (2009) presented a SPH variant by deriving a set of general discrete hydrodynamic equations within an energy-based framework. They highlighted that their formulations are also consistent with those derived from a variational approach by Bonet and Lok (1999). The connection in between variational principle and energy conservation in SPH has been well illustrated (e.g. Monaghan and Price 2001; Violeau 2012). Violeau (2012) highlighted the compatibility, and more precisely, the skew-adjointness of gradient and divergence operators for energy conservation. In the context of projection-based particle methods, this important property is required for an exact projection (Cummins and Rudman 1999) which is a necessity for an exact energy conservation.

Recently, Khayyer et al. (2015a) performed a study on energy conservation properties of projection-based particle methods, i.e. MPS and ISPH. Their study highlighted the significance of Taylor-series consistent pressure gradient models (e.g. Eqs. 8, 12) and enhancing effect of the consistency-related Gradient Correction (GC) scheme in providing enhanced energy conservation. 

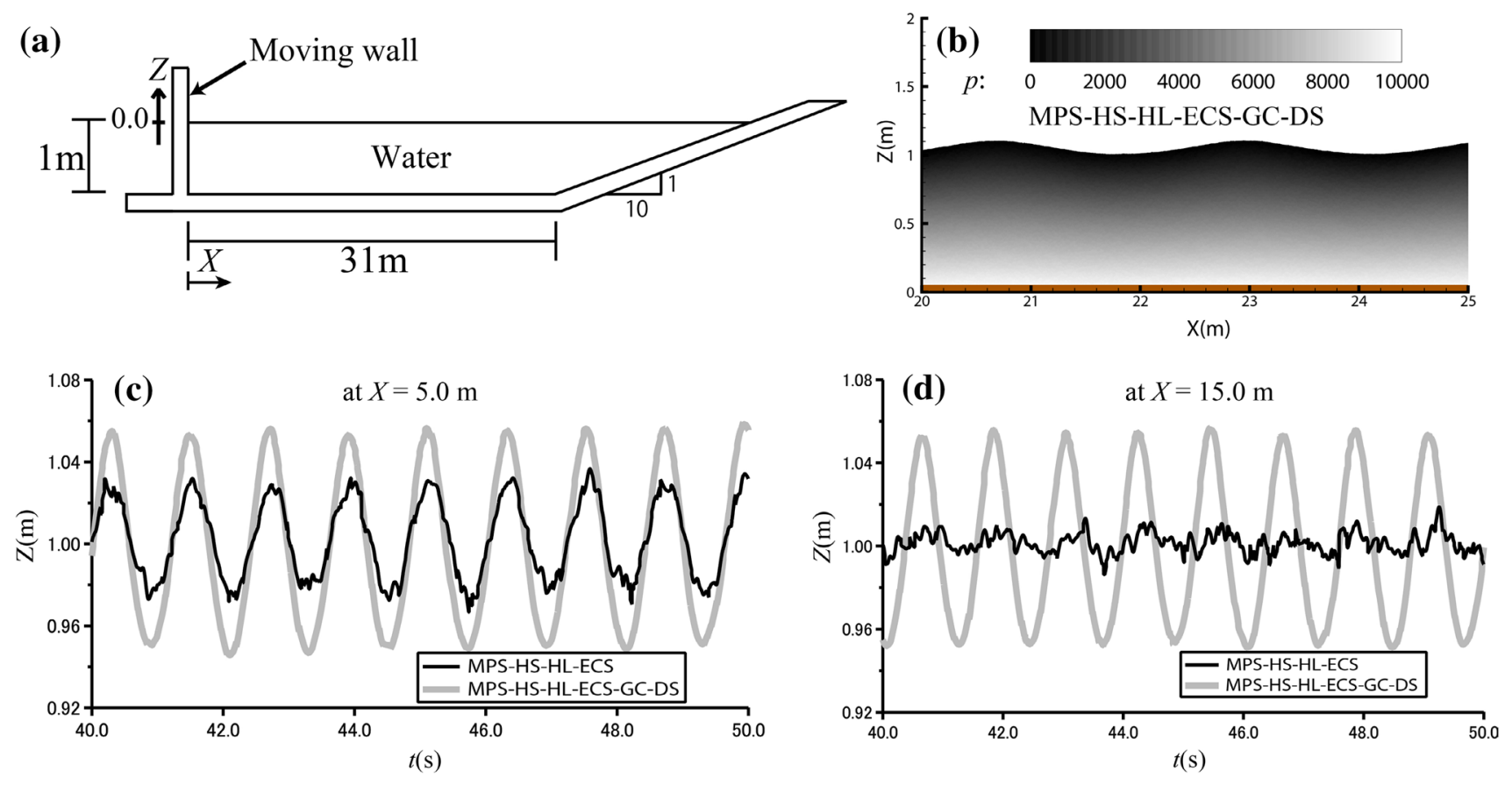

Fig. 2 Sinusoidal wave propagation on a long flat bottom-a schematic sketch of calculation domain, b typical snapshot of fluid particles together with pressure field, $\mathbf{c}$ time history of water elevation at $x=5.0 \mathrm{~m}$, and $\mathbf{d} x=15.0 \mathrm{~m}$

By applying Eq. 8 together with the refined schemes of HS, HL and ECS, enhanced energy conservations and acceptable predictions of physical dissipations could be achieved in the study by Khayyer et al. (2015a).

Figure 2 illustrates the appropriateness of Eq. 8 with respect to Eq. 11 in providing minimized numerical dissipation in a long-term wave propagation simulation. The considered wave is a sinusoidal one with wave period of 1.2 $\mathrm{s}$, wave height of $0.11 \mathrm{~m}$ and wave length of $2.1 \mathrm{~m}$. A total number of 383,815 particles were employed in the domain. The particle diameter, $d_{0}$, was considered to be $0.01 \mathrm{~m}$. Figure 2a shows a schematic sketch of calculation domain. Figure $2 \mathrm{~b}$ presents a typical snapshot of fluid particles together with calculated pressure field by the enhanced MPS incorporating Eq. 8 (MPS-GC-DS) together with HS, HL and ECS schemes so that the method is referred to as MPS-HS-HLECS-GC-DS. Quantitative comparisons of water elevations at horizontal positions of $x=5$ and $15 \mathrm{~m}$ are presented in Fig. 2c, d, respectively.

Figure 3 shows results of simulations of a standing wave by ISPH-based methods, where exactly similar tendency as MPS-based simulations could be observed. In other words, a Taylor-series consistent SPH pressure gradient model with a consistency-related correction has provided far better results compared with the symmetric repulsive pressure gradient model (Eq. 14). Conditions of the performed simulations shown in Fig. 3 correspond to those in Suzuki et al. (2007). In other words, the water depth $h$ is $1 \mathrm{~m}$ and the bottom width is $2 \mathrm{~m}$. Initial profile of water surface is given as follows:

$\eta_{0}(x)=A \cos [k(x+\lambda) / 2]$,

where $\eta_{0}$ is the initial surface elevation (above the mean water level at $y=1.0 \mathrm{~m}), A=0.1 \mathrm{~h}$ is the wave amplitude, $k=2 \pi / \lambda$ is the wave number and $\lambda(=2 \mathrm{~m})$ is the wave length. Initial velocity is set as zero for all the particles. The calculation time step is obtained based on the Courant stability condition and a maximum allowable time step of $\Delta t=2.5 \mathrm{E}-4 \mathrm{~s}$. The diameter of particles is set as $d_{0}=0.01 \mathrm{~m}$.

Figure 4 illustrates the improved MPS results of the normal impact of two rectangular fluid patches (Marrone et al. 2015). The rectangular patches have a length $L$, width $2 H$ and the impact occurs at $t=0$. The fluid is considered to be inviscid and incompressible, and thus the impact will be associated with a theoretically sudden loss of a fraction of the initial energy (Szymczak et al. 1994). For the performed simulations $L=1.0 \mathrm{~m}, H=0.33 \mathrm{~m}$ and $U=3.4 \mathrm{~m} / \mathrm{s}$. The maximum allowable time step is set as $\Delta t=5.0 \mathrm{E}-5 \mathrm{~s}$ and the particles are set to be of $0.01 \mathrm{~m}$ in diameter, i.e. $d_{0}=0.01 \mathrm{~m}$. A set of typical snapshots illustrating this phenomenon is presented in Fig. 4a-d. From Fig. 4e, the enhanced MPS method which benefits from five refined schemes, namely HS-HLECS-GC-DS has been able to properly reproduce this loss of energy. The MPS with a repulsive-based pressure gradient model (Eq. 11) has been inaccurate even by employment of 
Fig. 3 Simulations of a standing wave by ISPH-based methods with symmetric repulsive pressure gradient model (a) and Taylor-series consistent one (b)-quantitative comparison in terms of water level elevation at the center of the tank (c)

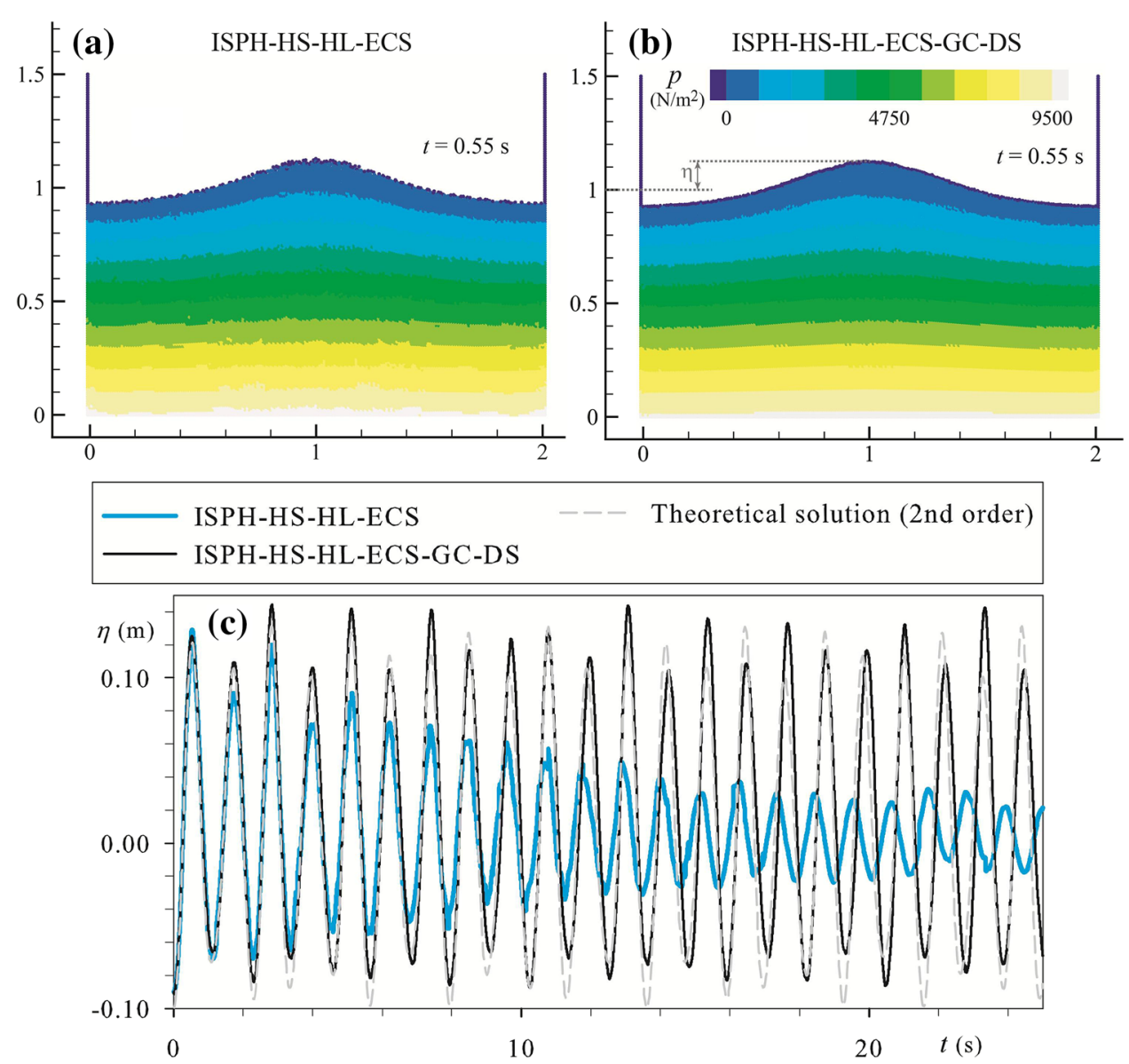

HS and HL schemes. The figure also portrays the enhancing effect of ECS scheme.

\subsection{Improvement of boundary conditions}

There are several important aspects to be considered for a proper enforcement of boundary conditions including solid, free-surface, inlet/outlet boundaries, etc. These issues as discussed by the SPHERIC boundary conditions' working group correspond to preservation of conservation and consistency properties of particle methods. In this section, we review the latest advancements made for treatment of solid (wall), free-surfaces as well as inlet/outlet boundaries.

\subsubsection{Solid or wall boundaries}

Non-conservative, inconsistent wall boundary conditions result in non-conservation of volume and momentum, and accordingly may either result in unphysical wall penetrations or gaps in between wall particles and fluid ones.

Treatment of solid wall boundaries in particle methods, and in particular in SPH, has been carried out mainly by the use of so-called ghost (Colagrossi and Landrini 2003) or mirror (Basa et al. 2009) particles as fictitious neighboring particles that are positioned to complete the truncated kernel supports at boundaries. By applying a pressure boundary condition founded on local force balance in between wall and fluid particles, Adami et al. (2012) proposed a generalized wall boundary condition for SPH which correctly imposes no-slip conditions even for complex geometries. Despite being relatively simple for implementation, application of mirror particles may lead to inaccuracies in the convergence of differential operator models (Macià et al. 2011). A more favored and recent approach is related to development of socalled semi-analytical wall boundary conditions.

Di Monaco et al. (2011) developed a semi-analytic approach for treatment of wall boundaries that can be considered as an integral version of the mirror particles of Adami et al. (2012) for fixed boundaries. Similar approaches have been proposed by Ferrand et al. (2013) and Mayrhofer et al. (2013) that provide accurate and direct modeling of boundary integrals at the frontiers of the fluid domain resulting in precise pressure forces, wall friction and turbulent conditions. The importance of proper modeling of boundary conditions by careful implementation of boundary integrals for accuracy, consistency and convergence of both weakly compressible SPH and ISPH was shown in a study by Macià et al. (2012). 
Fig. 4 The normal impact of two rectangular fluid patches a-d snapshots of particles together with pressure field, $\mathbf{e}$ time history of the evolution of mechanical energy

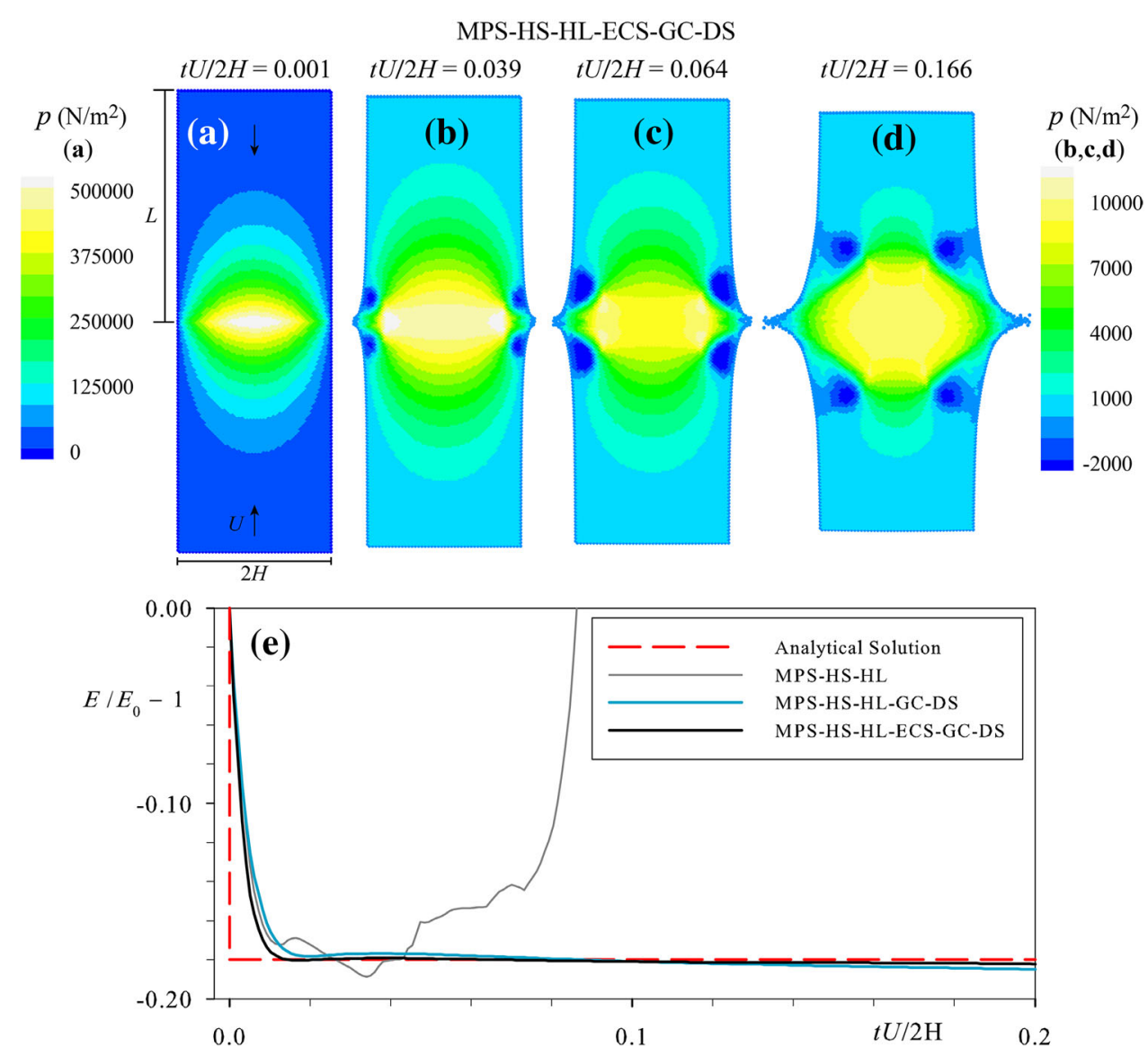

Another class of wall boundary conditions commonly applied in SPH corresponds to the repulsive boundary forces (Monaghan 2005) that may not properly model the actual physics in the vicinity of the boundary due to possible generation of excessive repulsive forces.

As for projection-based particle methods, uniformly spaced, fixed dummy particles are commonly applied to treat the wall boundaries (e.g. Gotoh and Sakai 1999; Shao and Lo 2003; Gotoh et al. 2005; Lee et al. 2008). In general, a few layers of dummy particles are added to provide a complete compact support for particles in the vicinity of wall boundaries, while only one or two layers of dummy particles are considered in the pressure solution process. For enhanced particle methods (e.g. Gotoh et al. 2014; Khayyer and Gotoh 2013) that provide acceptable pressure field and volume conservation, at least the pressure forces at the wall boundaries are calculated physically and precisely. Hence, the problem of unphysical wall penetration would become unlikely. On the other hand, for proper modeling of wall friction and turbulent conditions appropriate sub-models and careful considerations need to be taken into account.

It should be stated here that the concept of mirror particles has also been incorporated with projection-based particle methods such as the ISPH. For instance, Liu et al.
(2013) proposed an improved mirror particle treatment in their ISPH-based simulations of wave-structure interactions. An advantage of mirror particle technique with respect to the popular dummy particle approach (that assigns a zero velocity to all boundary particles) is that mirror particles theoretically impose the no-slip boundary condition more accurately as they are intrinsically founded on a linear extrapolation concept (Violeau 2012).

Recently, Leroy et al. (2014) extended the unified semianalytical wall boundary condition of Ferrand et al. (2013) for the projection-based particle methods, and more precisely, the ISPH method. The main feature of their work was the exact enforcement of a non-homogeneous Neumann boundary condition on the pressure field that resulted in a distinct form of PPE. The ISPH model of Leroy et al. (2014) was further extended to buoyancy modeling for both laminar and turbulent flows (Leroy et al. 2015a) where buoyancy effects were modeled through the coupling of Boussinesq approximation and a heat equation.

\subsubsection{Free-surface boundary condition}

In projection-based particle methods, a challenging issue is to detect free-surface particles accurately to impose the 


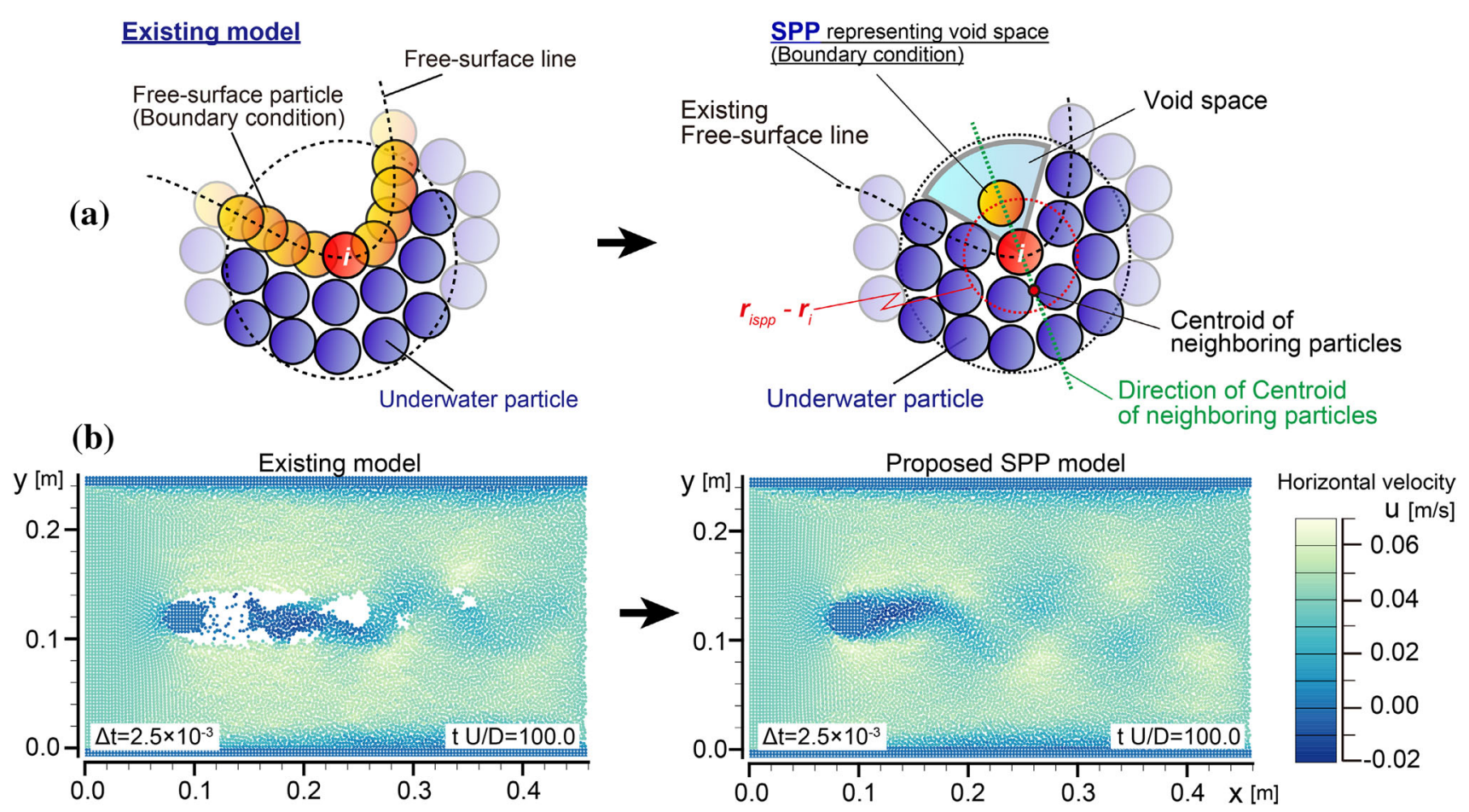

Fig. 5 A graphical representation of the SPP (space potential particles) scheme (a), effectiveness of SPP in elimination of unphysical voids in a Karman vortex simulation (b) (Tsuruta et al. 2015)

dynamic free-surface boundary condition, i.e. $p$ equal to zero, on them. This would also be an important condition in the solution process of the PPE. There have been several freesurface detection techniques including the simplest scheme founded on the fact that particle number density sharply drops at the free-surface (e.g. Koshizuka and Oka 1996; Shao and Lo 2003; Gotoh and Sakai 2006). Lee et al. (2008) used a property corresponding to divergence of particle positions to detect the free-surface particles. Khayyer et al. (2009) proposed an auxiliary condition based on the non-symmetric distribution of free-surface particles to be used together with the original simple criterion. Ma and Zhou (2009) proposed a mixed particle number density and auxiliary function method (MPAM) for identifying the free surface particles in their Meshless local Petrov-Galerin method based on Rankine source solution (MLPG-R) method. Park et al. (2014) used a so-called Arc Method for an accurate assessment of freesurface particles.

Skillen et al. (2013) proposed a new idea of gradually introducing the effect of discontinuous free-surface with the aim of minimizing the temporal pressure noise. Nair and Tomar (2014) presented a semi-analytical approach to impose Dirichlet boundary conditions on the free surface and, therefore, the need for free-surface particle assessment was eliminated in their study. This necessity was also eliminated by proposal of a new free-surface boundary condition referred to as Space Potential Particles (SPP; Tsuruta et al. 2015) and through introduction of a potential in void space to reproduce physical motions of particle around freesurface through a particle-void interaction. Figure 5 shows a graphical representation of the SPP scheme as well as its effectiveness in elimination of unphysical voids in a Karman vortex simulation corresponding to a Reynolds number of 1200. Details of this simulation are given by Tsuruta et al. (2015). For this Karman vortex simulation the calculation domain is set as a channel with $0.45 \mathrm{~m}$ length and $0.24 \mathrm{~m}$ width. A cylinder with a diameter of $0.3 \mathrm{~m}$ was set at a position of $(x, y)$ being $(0.09 \mathrm{~m}, 0.12 \mathrm{~m})$. The water particles were considered to be $3 \mathrm{~mm}$ in diameter.

\subsubsection{Inlet/outlet boundary conditions}

The most crucial and challenging issue in implementation of inlet/outlet boundary conditions corresponds to accurate enforcement of mass (or volume) conservation. There have been a number of researches specifically targeting inlet/outlet boundary conditions in both weakly compressible (e.g. Lastiwka et al. 2009) and incompressible (e.g. Khorasanizade and Sousa 2016) frameworks.

In order to enhance the ISPH solution for both pressure and velocity near the boundaries including inlet/outlet ones, Hosseini and Feng (2011) presented an approach which utilizes a rotational pressure-correction scheme with a consistent pressure boundary condition. Shibata et al. (2011) 
presented a so-called transparent boundary condition for an accurate absorption of Stokes wave at absorbing boundaries. Liu et al. (2015) presented a non-reflection internal wave maker for the ISPH method. A novel formulation for inflow-outflow boundary conditions in an ISPH framework is recently proposed by Leroy et al. (2015b). This formulation is founded on the unified semi-analytical technique proposed for treatment of wall boundary conditions (Ferrand et al. 2013) and extended to open boundaries (Kassiotis et al. 2013) in WCSPH framework.

\subsection{Multi-phase flows}

A key challenge in particle-based simulations of multiphase flows, especially those characterized by large density ratios corresponds to the sharp and abrupt density drop at the phase interface that would lead to a mathematical discontinuity of density and accordingly a discontinuous pressure gradient field. Thus, even slight inaccuracies in pressure gradient calculation would bring about numerical instabilities that may end up in a complete blow-up of simulation.

In the context of particle methods, there have been several attempts to propose stable and accurate multiphase methods that can deal with the mathematical discontinuity of density at the phase interfaces. The so-far conducted researches conducted in the framework of weakly compressible (or fully explicit) particle methods can be categorized into the following three distinct groups:

(i) Density evaluation through a spatial averaging: The most common approach is to calculate the densities at target particles by performing a proper spatial weighted averaging through the implementation of a corrected kernel (e.g. Colagrossi and Landrini 2003; Grenier et al. 2009).

In addition to applying a spatially averaged density, the SPH-based multiphase simulations of Colagrossi and Landrini (2003) and Grenier et al. (2009) were carried out by use of some sort of numerical stabilizers, e.g. an unphysical surface tension term (as in Colagrossi and Landrini 2003) or an unphysical repulsive pressure force between particles of different fluids (as in Grenier et al. 2009). Despite improving the stability and minimizing the numerical dispersions at the phase interfaces, such unphysical forces will result in an unphysical gap in between the fluids of different phases. Shakibaeinia and Jin (2012) applied a modified version of a so-called weakly compressible MPS to simulation of multiphase flows with low-density ratios. The modifications comprised of density and viscosity smoothening schemes, and more precisely, application of the simplest possible spatial averaging of density (corresponding to a zeroth-order accurate SPH scheme) and a harmonic mean for viscosity. Despite being helpful in dealing with the mathematical discontinuity of density at a phase interface, the considered scheme by Shakibaeinia and Jin (2012) simply results in an unphysical diffusion and accordingly an unphysical smoothening of density as well as unphysical dispersions of fluid particles at the phase interfaces.

(ii) Lagrangian equations: Monaghan and Rafiee (213) proposed a robust SPH algorithm based on the Lagrangian equations and successfully simulated several multiphase flows with high-density ratios without a density smoothening scheme. Nevertheless, their simulations were performed using a repulsive pressure force between particles of different fluids as well as an artificial viscosity term. Further, in some cases, simulation results of Monaghan and Rafiee (213) showed unphysical perturbations at the phase interfaces.

(iii) Energy density-based smoothing: Saitoh and Makino (2013) developed an alternative SPH model which incorporates energy density, rather than mass density, as the basis for smoothing. By utilizing this approach, density differentiability is no longer a prerequisite and hence contact discontinuities can be handled efficiently. Nevertheless, the simulation results of Saitoh and Makino (2013) are still characterized by notable numerical diffusion of density resulting in an unphysical smoothening of the interface sharpness.

As for projection-based particle methods, the so-far developed methods either consider a special treatment at the phase interface or tend to use a combined grid-based and gridless approach.

(i) Consideration of an interactive force at the phase interface: Ikari et al. (2004) were the first to propose a gas-liquid two-phase MPS method by treating the gas and liquid phases as discrete particles and considering an interaction force in between them. To assure the stability of their calculations, they defined a specific procedure to maintain the gas particles in adequate distances from the liquid particles by introducing interacting repulsive forces. The multiphase MPS methods of Ikari et al. (2004) was verified mainly qualitatively and solely by coastal engineering related applications. Shao (2012) proposed a decoupled ISPH method through a special treatment of interface particles and consideration of particular interface boundary conditions to tackle the discontinuity of density at the phase interface in their multi-fluid simulations.

(ii) Hybrid particle-mesh methods: In order to deal with the mathematical discontinuity of density, Liu et al. (2005) proposed a hybrid MPS-FVM particle-mesh method where the heavier phase was represented by particles 
Fig. 6 Typical snapshots corresponding to a multiphase violent flow sloshing characterized by air entrainment/entrapment illustrating the effectiveness of FDS scheme-snapshots of air/water particles $(\mathbf{a}, \mathbf{b})$ and snapshots of particle together with density field $(\mathbf{c}, \mathbf{d})$ (Khayyer and Gotoh 2013)
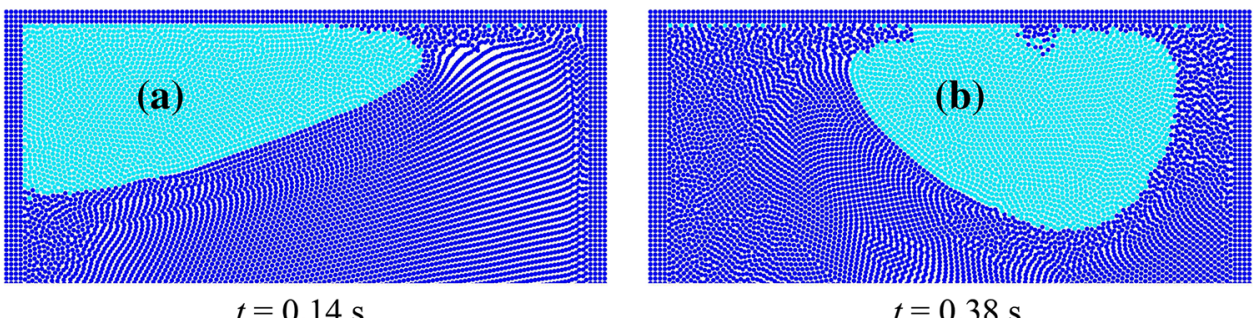

$t=0.14 \mathrm{~s}$

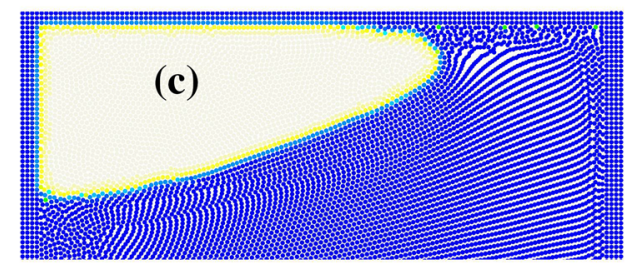

$$
t=0.38 \mathrm{~s}
$$

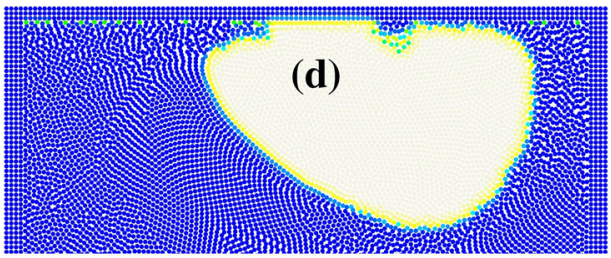

MPS-HS-HL-ECS-GC-FDS

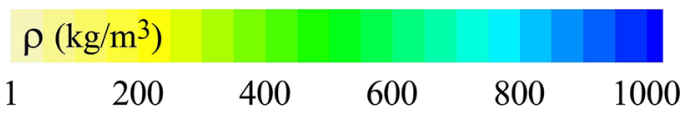

and the lighter one was defined on a mesh. The discontinuities were resolved by extrapolating the density and viscosity of interfacial particles onto the mesh.

(iii) Application of particle shifting technique: In order to deal with particle non-uniformity issues at the phase interface, Lind et al. (2015) applied the particle shifting technique (Lind et al. 2012) in their incompressiblecompressible SPH simulations of water-air wave slamming through a proper coupling of a weakly compressible SPH for the gas phase and an incompressible SPH for the liquid one. The effectiveness of the particle shifting technique was further illustrated in the recent paper by Lind et al. (2016) where a thoroughly validated compressible-incompressible SPH was presented. The proposed multiphase SPH method of Lind et al. (2016) was shown to maintain a true material discontinuity at the phase interface together with physically correct and continuous pressure/velocity fields.

(iv) Density evaluation through a spatial averaging: To deal with the discontinuity of density, $\mathrm{Hu}$ and Adams (2006) reformulated their incompressible SPH schemes by considering the so-called "particle number density", consistent with MPS descriptions, that resulted in a continuous form of pressure gradient formulation.

Khayyer and Gotoh (2013) presented an improved MPS method for multiphase flows characterized by large density ratios. The stability of their calculations was guaranteed through the application of a first-order-accurate Taylorseries-based Density Smoothing (FDS) scheme, and accuracy enhancement was achieved through the application of a PPE's error mitigating term (ECS) and refined discretizations of source term (HS) and Laplacian of pressure (HL). The FDS scheme was shown to provide significantly improved results with respect to the ZDS (Zeroth-order accurate Density Smoothing) one. Figure 6 presents two typical snapshots corresponding to a violent sloshing flow with reproduced distributions of gas-liquid particles $(a, b)$ as well as density field (c, d) by an enhanced multiphase MPS incorporating the FDS scheme. Conditions of the performed sloshing simulation corresponded to the experiment by Rognebakke et al. (2006). Sinusoidal excitations with maximum amplitude of $150 \mathrm{~mm}$ and frequency of $1.2 \mathrm{~Hz}$ were considered. The particles were $5.0 \mathrm{~mm}$ in diameter and the calculation time step was set according to the Courant stability condition and a maximum allowable time increment of $4.0 \mathrm{E}-5 \mathrm{~s}$.

Recently, Khayyer and Gotoh (2016) extended their ECS scheme to minimize the projection-related errors in an airwater compressible-incompressible multiphase calculation of wave slamming. The extended ECS was referred to as CIECS (compressible incompressible ECS). For their calculations, Khayyer and Gotoh (2016) considered an integrated set of equations for the gas and liquid phases with compressible forms of continuity equations and by implementations of actual speeds of sounds in air and water. Figure 7 shows a set of results corresponding to this study. Figure $7 \mathrm{a}-\mathrm{c}$ depicts the water slamming simulation results related to the experiment by Lin and Shieh (1997) by multiphase and single-phase MPS methods. The figure highlights the importance of consideration of air and its cushioning effect for prediction of slamming-induced pressures. Figure $7 d$ portrays a comparison in between the multiphase MPS with CIECS scheme with results by Lind et al. (2015) and Ma et al. (2014) with respect to the experiment by Verhagen (1967). A common experiment-simulation inconsistency seen in this figure corresponds to inaccurate prediction of post-impact negative pressure. In the performed water slamming simulations, the diameter of particles was set as $3 \mathrm{~mm}$. Considered viscosities 

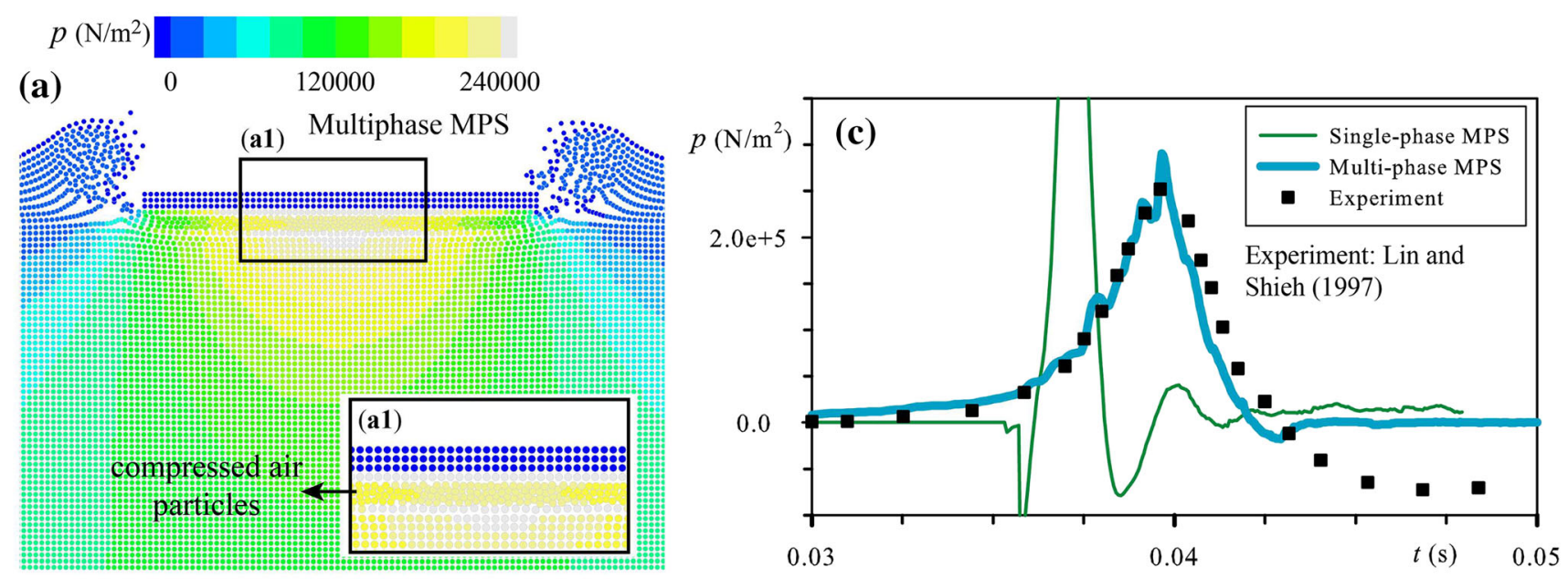

(b) Single-phase MPS
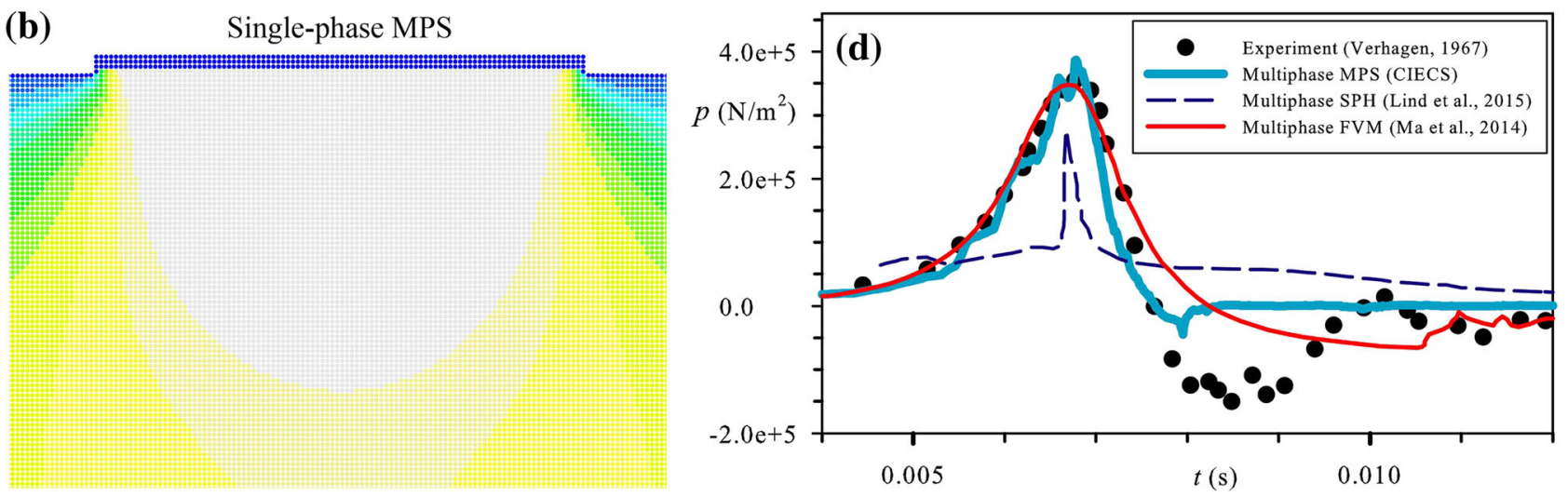

Fig. 7 Typical snapshots illustrating a water slamming corresponding to the experiment by Lin and Shieh (1997) by an enhanced multiphase MPS (a) and an enhanced single-phase MPS (b)-time history of pressure at the center of the plate (c)-quantitative comparison of

slamming induced pressure at the center of a plate corresponding to the experiment by Verhagen (1967), results by enhanced multiphase MPS, multiphase SPH (Lind et al. 2015) and multiphase FVM (Ma et al. 2014) (d) (Khayyer and Gotoh 2016)

for the water and air phases corresponded to their physics ones, i.e. $v_{w}=1.0 \mathrm{E}-6 \mathrm{~m}^{2} / \mathrm{s}$ and $v_{\mathrm{a}}=1.5 \mathrm{E}-5 \mathrm{~m}^{2} / \mathrm{s}$. The calculation time step was set based on the Courant stability condition and $\Delta t_{\max }=1.0 \mathrm{E}-4 \mathrm{~s}$.

Indeed, the multiphase simulations by particle methods are not limited to only liquid-gas simulations, but also solid-liquid simulations (e.g. Gotoh and Sakai 2006). The pioneering work related to multiphase flow simulations by projection-based particle methods corresponds to that by Gotoh and Fredsøe (2000) who developed a solid-liquid twophase MPS method. A number of interesting ocean or coastal engineering applications have been studied by solid-liquid MPS methods as illustrated by Gotoh and Sakai (2006).

\subsection{Surface tension}

Consideration of surface tension becomes important when deformations of fluid surfaces are involved. For example, surface tension plays a key role in splash generation due to finger-jet break-up at the tip of the wave-breaking jet. The splash generation drastically increases the surface area of water drops which enhances gas exchange between atmosphere and seawater. Another example corresponds to the later phases of the spreading of oil in water which is driven by surface tension forces. Thus, surface tension modeling is of significant importance in ocean/offshore engineering.

The approaches applied for modeling surface tension in macroscopic particle-based methods can be divided into two main categories, namely, the so-called potential approach and the so-called continuum approach.

\subsubsection{Potential approach}

In this approach the surface tension is modeled by assuming that microscopic cohesive intermolecular forces can be mimicked by macroscopic inter-particle forces. The main advantage of this approach corresponds to its computational simplicity in that surface tension is modeled via 
particle-particle interactions explicitly without the necessity of calculating surface normals and curvatures as required in the continuum approach. The major disadvantage of potential approach is related to the fact that the surface tension forces depend on the intensity of particle-particle interactions. These interactions have to be adjusted numerically by varying the macroscopic input parameters depending on the simulation case to reproduce desired surface tension forces. Thus, this approach is not preferable from the practical engineering viewpoint unless the considered particle method is extended to micro/nano scales. It is also worth mentioning that with given parameters, the potential-based surface tension modeling approach is resolution dependent and the modeled surface tension does not converge to a fixed value with refinement of resolution (Adami et al. 2010)

A number of potential-based surface tension modeling exist in the field of particle method research. Nugent and Posch (2000) applied cohesive van der Waals type potentials between two fluids in their multi-phase calculation of 2D liquid drop condensations. They highlighted the fact that for stable simulations the interaction range of particle interactions should be about twice of that of SPH smoothing length, which results in a less computationally efficient calculation. Tartakovsky and Meakin (2005) utilized a similar approach but instead of van der Waals type interactions, a combination of attractive and repulsive forces was considered within the range of standard SPH kernels. Due to its simplicity, several multiphase SPH calculations including those related to flows in porous media (e.g. Alvarado-Rodriguez et al. 2015) have been founded on this approach. Recently, Tartakovsky and Panchenko (2016) proposed an updated molecular-like Pairwise Force-SPH model for incorporation of surface tension and contact line dynamics. Their model is characterized by new approximate relationships between the molecular-like forces and macroscopic properties of a multiphase flow.

In the field of MPS research, Shirakawa et al. (1999) presented the first potential-based surface tension modeling similar to molecular dynamics approach. In general the considered potential functions have been proposed by either discontinuous (e.g. Shirakawa et al. 1999) or continuous (e.g. Kondo et al. 2007) functions. Several studies have been devoted to proposal of an appropriate potential function for a more reliable simulation of surface tension (e.g. Ishii and Kohira 2009; Ishii and Sugii 2011; Natsui et al. 2012).

Another computational issue corresponding to potential approach is related to probable occurrence of numerical instability, especially when the particles are not regularly distributed (Zhang et al. 2008). Different smoothing procedures have been proposed to tackle this problematic issue (e.g. Zhang et al. 2008; Ishii and Sugii 2011).

\subsubsection{Continuum approach}

The most common approach for incorporation of surface tension in macroscopic particle-based simulations is based on the continuum surface force (CSF) model introduced by Brackbill et al. (1992). In this approach, the surface tension is treated as a continuous, three-dimensional effect across the interface, derived directly from the Young-Laplace equation:

$\Delta p=-\sigma \operatorname{div}(\boldsymbol{n})$,

where $\Delta p$ is the pressure jump across the interface, $\sigma$ is the surface tension coefficient and $\boldsymbol{n}$ is the interface normal pointing out towards the gas phase. This pressure jump is applied via a volume force normal to the interface:

$\boldsymbol{F}=-\sigma \kappa \boldsymbol{n} \delta_{s}$,

where $\kappa$ is the average curvature which is obtained by taking the divergence of the normal vector $(\kappa=-\nabla \cdot \boldsymbol{n})$ and $\delta_{s}$ stands for the surface-delta function. In order to approximate the characteristics of the interface, i.e. normal direction and curvature, a volume fraction function, usually referred to as color function $C$, is defined. The normal vector $\boldsymbol{n}$ is determined as the normalized gradient of this color function, i.e. $\boldsymbol{n}=\nabla C /|\nabla C|$.

Morris (2000) showed several possible implementations of CSF model in SPH, both with and without exact conservation of momentum, and highlighted the challenges in accurate calculations of interface curvature. These challenges are not only limited to difficulties in accurate particle-based calculation of Laplacian of color function for approximation of interface curvature, but also to the fact that a smoothed color function is usually used. The use of a smoothed color function may become problematic for approximation of interface normals near the boundaries and sharp-angled areas. Recently, Duan et al. (2015) proposed a so-called CCSF (contoured continuum surface force) model characterized by a cumbersome analytical calculation of interface curvature based on a locally constructed smoothed color function within the MPS framework. The authors applied a well-known formulation applied in Eulerian Level Set methods by considering contours of a smoothed color function to obtain an estimation of interface curvature. In addition to the presence of complexity, the accuracy of their proposed method appeared to be dependent upon the choice of smoothing radius, while this is not the case in well-known established SPH surface tension models.

In order to resolve these two challenging issues, $\mathrm{Hu}$ and Adams (2006) presented a continuous surface stress model (CSS) using a discontinuous, sharp color function to directly calculate the pressure jump from the interface stress tensor and modeled the surface tension in a more accurate and momentum conservative manner. In their model, calculation 
of surface curvature was avoided due to consideration of a surface stress tensor. Further, since the magnitude of this tensor is proportional to that of the color gradient, the contribution of a small color gradient at the edge of transition bands does not bring about numerical difficulties (Adami et al. 2010).

A set of efforts has been focused on enhancing the particlebased CSF model by providing more accurate schemes for approximation of interface normal and curvature. For instance, Adami et al. (2010) proposed a new formulation for the surface curvature by applying a reproducing divergence approximation. Qiang et al. (2011) applied a Taylor-series based correction leading to more accurate interface normals and thus curvatures.

In the field of MPS research, the CSF-based simulations can be categorized into two distinct groups, depending on the computational procedure for calculation of the curvature and the normal vector. These two categories are: arc fitting at interface (Nomura et al. 2001) and differential approach (e.g. Ichikawa and Labrosse 2010).

\subsubsection{Arc fitting at interface}

As the name indicates the arc fitting approach is aimed at approximating the normal vector and curvature by constructing local arcs at the surface particles. To achieve this approximation, a layer of fluid particles at the interface, with a thickness of $d_{\text {st }}$, is considered as free-surface for which surface tension forces are to be calculated. Specific particle number densities including an initial one and a revised one are calculated to approximate the curvature and the unit normal. Despite its simple and comprehensible algorithm, the accuracy of this approach is highly dependent upon the instantaneous smoothness of the free-surface. This fact is highlighted by Gotoh et al. $(2004,2005)$ and Ikari et al. (2004). Furthermore, even in the presence of a smooth free-surface, accomplishment of a continuous curvature calculation would be difficult, in both time and spatial domain. This is due to the calculation of curvature and normal vectors by discrete values of particle number densities as well as simple finite difference schemes for evaluation of normal vectors. It should be noted that despite these deficiencies, improved models for approximation of the unit normal can be obtained by applying higher order finite difference approximations. For instance, Rong and Chen (2010) applied a fourth-order central differencing scheme to approximate the derivatives used for the unit normal vector.

\subsubsection{Differential approach}

The favored approach for modeling surface tension by particle methods, including projection-based ones, is to calculate the continuum surface forces by applying consistent and accurate differential operator models for both gradient and Laplacian so that accurate approximations of the unit normal vector and the curvature can be obtained. Shirakawa et al. (1999) were among the first who illustrated possible development of a differential CSF-based surface tension model for MPS. However, they pointed out that this approach is not preferable due to the difficulty of curvature evaluation at a free-surface cusp. Liu et al. (2005) and Zhang et al. (2007) applied differential CSF-based surface tension modeling in their hybrid particle methods. Alam et al. (2007) applied this approach for surface tension modeling in their MPS simulations of water splash phenomena.

In most cases, the evaluation of normal vector was conduced by use of original MPS gradient model, while the curvature was obtained by applying the original MPS divergence model to the approximated unit normal vector, illustrating that the curvature calculation is obtained by a simplified approximation based on approximated values. This would highlight the need to enhance the accuracy of unit normal vector calculation beforehand if the curvature model would be directly dependent upon the approximated unit normal vector. In an attempt to improve the accuracy of differential CSF-based surface tension modeling in MPS, Ichikawa and Labrosse (2010) applied a SPH-based scheme to evaluate the unit normal vector, yet the curvature was found by the original MPS divergence model.

By conducting a simple comparison, Park and Jeun (2011a) showed that approximation of normal vector by a differential operator model is more accurate than that by a socalled four-point technique as used in arc fitting approach. A differential CSF-based model was also used by these authors in their isothermal multiphase MPS calculations (Park and Jeun 2011b). Khayyer et al. (2014) proposed a new differential CSF-based model in the context of MPS. Their model benefits from a novel formulation for curvature estimation using direct second-order derivatives of color function via a meticulous and comprehensive discretization. By applying a high-order Laplacian scheme including the approximation of boundary integrals, relatively accurate approximation of interface curvature and thus surface tension could be achieved. In the work by Khayyer et al. (2014), the Laplacian of color function, $C$, at an interface target particle $i$ is calculated as

$$
\begin{aligned}
\left(\nabla^{2} C\right)_{i}= & \frac{1}{n_{0}} \sum_{i \neq j}\left\{\frac{\partial C_{i j}}{\partial r_{i j}} \frac{\partial w_{i j}}{\partial r_{i j}}+C_{i j}\left(\frac{\partial^{2} w_{i j}}{\partial r_{i j}^{2}}+\frac{D_{s}-1}{r_{i j}} \frac{\partial w_{i j}}{\partial r_{i j}}\right)\right\} \\
& +\mathrm{BI}
\end{aligned}
$$

where $C_{i j}=C_{j}-C_{i}$ and BI denotes the boundary integrals (Souto-Iglesias et al. 2013) formulated as

$$
\mathrm{BI}=\int_{\partial \Omega} \nabla C \cdot \boldsymbol{n} w\left(\left|\boldsymbol{r}_{i j}\right|\right) \mathrm{d} S \approx \frac{1}{n_{0}} \sum_{j \in \partial \Omega} \frac{C_{i j} \boldsymbol{r}_{i j} \cdot \boldsymbol{n}_{j}}{\left|\boldsymbol{r}_{i j}\right|^{2}} w\left(\left|\boldsymbol{r}_{i j}\right|\right) S_{j},
$$


Laplacian-based model
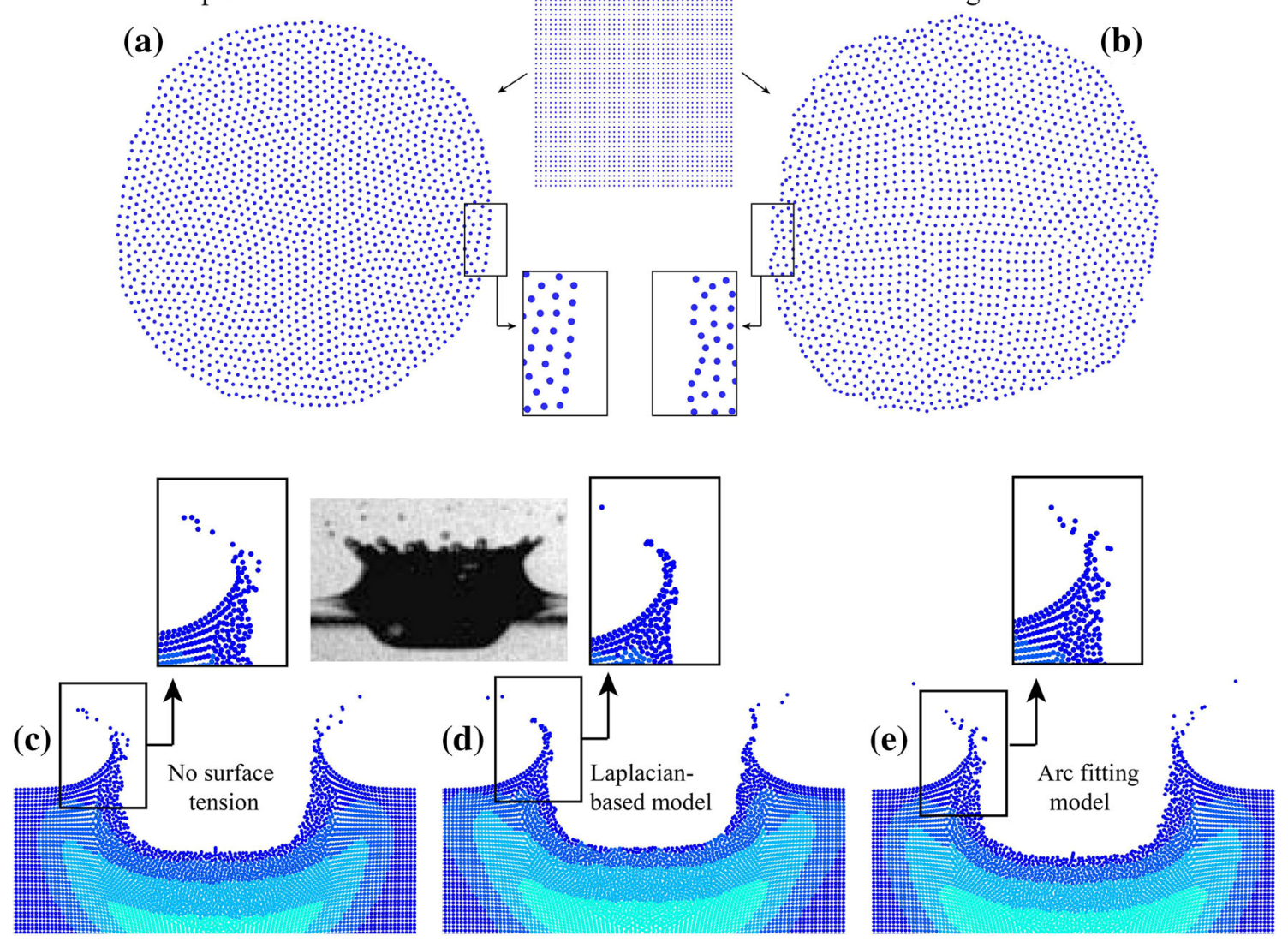

Fig. 8 Enhanced modeling of surface tension forces by a Laplacian-based model-typical snapshots corresponding to a non-equilibrium rod (a, b), and a water drop impact corresponding to the experiment by Liow (2001) (c-e)

where for 2D simulations, $S_{j}$ signifies the length (diameter) of boundary particle $j$. Therefore, the surface tension force is evaluated via achieving a direct Laplacian-based approximation of curvature.

The enhanced performance of the abovementioned Laplacian-based surface tension model with respect to the arc fitting one (Nomura et al. 2001) is illustrated in Fig. 8, corresponding to simulations of a non-equilibrium $\operatorname{rod}(\mathrm{a}, \mathrm{b})$ and water drop impact (c-e). The non-equilibrium rod corresponds to oscillation of an initially square drop under the action of surface tension forces. The initial square is an inviscid liquid with a diameter of $D=4 \mathrm{~mm}$, density of $\rho=1000$ $\mathrm{kg} / \mathrm{m}^{3}$ and surface tension coefficient of $\sigma=0.10 \mathrm{~N} / \mathrm{m}$. The particle size is considered to be $0.1 \mathrm{~mm}$. Due to the initial square shape of drop with theoretically infinite surface tension forces at the corners, the drop is set into oscillations towards an equilibrium circular shape. The Laplacian-based surface tension model has been able to reproduce an almost circular drop characterized by a smooth free-surface.

The snapshots shown in Fig. 8c-e correspond to the water drop impact experiments by Liow (2001), for Froude and Weber numbers of 639 and 395, respectively. The figure portrays the properness of Laplacian-based surface tension model in better reproduction of crown development and splash drops.

In another recent work, a differential CSF-based model was incorporated by Tiwari et al. (2016) for computation of surface tension in two-phase flows driven by wetting effects. The MLS (moving least square) method was used in that study for approximation of differential operators at each target particle based on the information at neighboring particles.

\subsection{Fluid-structure interactions}

Many problems in ocean engineering involve fluid-structure interaction (FSI) processes where the flow field is altered by the encountered structures and their simultaneous responses to the hydrodynamic loads. Examples include tsunami impact on coastal/offshore structures, sloshing in LNG tanks with elastic baffles and wave interactions with floating bodies. Hence, accurate simulation of FSI problems including proper resolutions of instantaneous flow field and structural response should be of significant importance in ocean engineering.

Despite their significant importance, FSI problems encountered in ocean engineering are challenging to analyze due to the presence of violent free-surface flows induc- 
ing large/abrupt hydrodynamic loads and thus considerable structural responses, possibly leading to large structural deformations and/or structural failures. From mathematicalnumerical viewpoint, existence of multi-domain characteristics and interface coupling conditions will further add to the existing complexities.

In the context of FSI simulations, particle methods including projection-based ones appear to be suitable computational tools. These methods have been applied to simulate interactions in between fluid flows with either rigid (e.g. Liu et al. 2013) or flexible (e.g. Rafiee and Thiagarajan 2009) structures. In the latter case, a proper structural model should be carefully coupled with the fluid solver.

In the field of particle methods, Libersky et al. (1993) and Gray et al. (2001) applied the SPH method to dynamic problems of elastic body. Antoci et al. (2007) and Oger et al. (2010) applied the SPH method for fully Lagrangian simulations of FSI problems involving weakly compressible flows interacting with deformable elastic structures. Yang et al. (2012) proposed a coupled weakly compressible SPH-FEM (finite element method) solver for FSI problems related to elastic structures.

In the framework of projection-based particle methods, Lee et al. (2007) developed a MPS-FEM coupled method to study incompressible fluid flow interactions with elastic structures. Rafiee and Thiagarajan (2009) proposed a fully Lagrangian SPH-based solver for simulation of incompressible fluid-hypoelastic structure interactions. In their study, the PPE was solved simply using an approximate explicit scheme. Hwang et al. (2014) developed a fully Lagrangian MPS-based FSI analysis method for incompressible fluidlinear elastic structure interactions. The key feature of the numerical method of Hwang et al. (2014) corresponded to its being free of any numerical stabilizing terms with calibration constants commonly applied in other particlebased FSI solvers. Such artificial stabilizing terms have been used in forms of artificial viscosity, artificial stress term or collision models to mainly deal with the tensile instability for both fluid and structure simulations in both SPH (e.g. Monaghan 1994; Antoci et al. 2007; Amanifard et al. 2011) and MPS (e.g. Lee et al. 213; Kondo et al. 2010; Shao et al. 2013) frameworks. This key feature of the study by Hwang et al. (2014) was achieved through application of a proper coupling algorithm and by taking the advantage of prediction-correction solution process of MPS as a projection-based method.

Khayyer et al. (2015b) presented a further enhanced version of Hwang et al.'s method, by incorporating the ECS and DS schemes for the fluid phase as well as applying a Wendland kernel (Wendland 1995; Dehnen and Aly 2012) for calculation of fluid forces on structure. The achieved enhancements as well as applicability of developed MPSbased FSI solver are illustrated in Figs. 9 and 10, correspond- ing to simulations of an entry of a deformable beam into an undisturbed water and a dam break flow impacting on an elastic plate.

Figure 9a illustrates an schematic sketch of the deformable beam entry test, where an aluminum beam enters an undisturbed water with a constant velocity of $30 \mathrm{~m} / \mathrm{s}$. A qualitative comparison in between the coupled MPS-based FSI solver and its enhanced version is presented in Fig. 9b, c. The snapshots depict the pressure and stress fields in fluid and beam, respectively. The snapshots by enhanced method tend to be characterized by improved and almost symmetric pressure fields. A quantitative comparison in terms of time histories of deflection at point $\mathrm{C}$ and time histories of pressure at point D is provided in Fig. 9d, e. From Fig. 9d, the enhanced FSI solver is found to provide a more accurate time history of deflection at point $\mathrm{C}$, quite consistent with the analytical solution (Scolan 2004) as well as a refined coupled SPH solver (Oger et al. 2010). Focusing on Fig. 9e, the enhanced coupled MPS solver has resulted in a more acceptable pressure time history compared with the coupled MPS as well as coupled SPH solvers. For this aluminum beam entry test, the analytical solutions were derived by Scolan (2004), on the basis of the hydrodynamic Wagner's model and linear Wan's theory. The material properties of the aluminum beam, namely its Young's modulus, Poisson ratio and density were considered as $67.5 \mathrm{GPa}, 0.34$ and $2700 \mathrm{~kg} / \mathrm{m}^{3}$, respectively. Both structural and fluid particles were $0.01 \mathrm{~m}$ in size.

Figure 10 corresponds to a dam break simulation with an elastic plate related to the experimental study of Liao et al. (2014, 2015). A schematic sketch of calculation domain as well as simulation conditions is presented in Fig. 10a. Both structural and fluid particles are considered to be $0.001 \mathrm{~m}$ in size. Figure $10 \mathrm{~b}-\mathrm{e}$ portrays a set of typical snapshots by coupled MPS (b,d) and enhanced coupled MPS (c,e) solvers together with their corresponding experimental photos as well as results by a FDM-FEM solver (Liao et al. 2014). The superior performance of enhanced MPS is clearly illustrated in this figure as this method provides more consistent deflections of the elastic plate both at $t=0.35 \mathrm{~s}$ and $t=0.39 \mathrm{~s}$. In particular, at $t=0.35 \mathrm{~s}$, there appears to be a non-physical separation of plate from the main incoming flow. At $t=0.39$ $\mathrm{s}$, the enhanced coupled MPS has been able to better reproduce the overall shape of the plate with a clear inflection point.

\subsection{Enhancement of computational efficiency}

A challenging issue corresponding to particle methods is related to their relatively high computational cost. Until recently, the high computational cost of particle methods was hindering their application to real-life problems, including large-scale scientific and engineering ones. The implementation of particle method codes on massively par- 


\section{Coupled MPS-based FSI Solver}

Hwang et al. (2014)
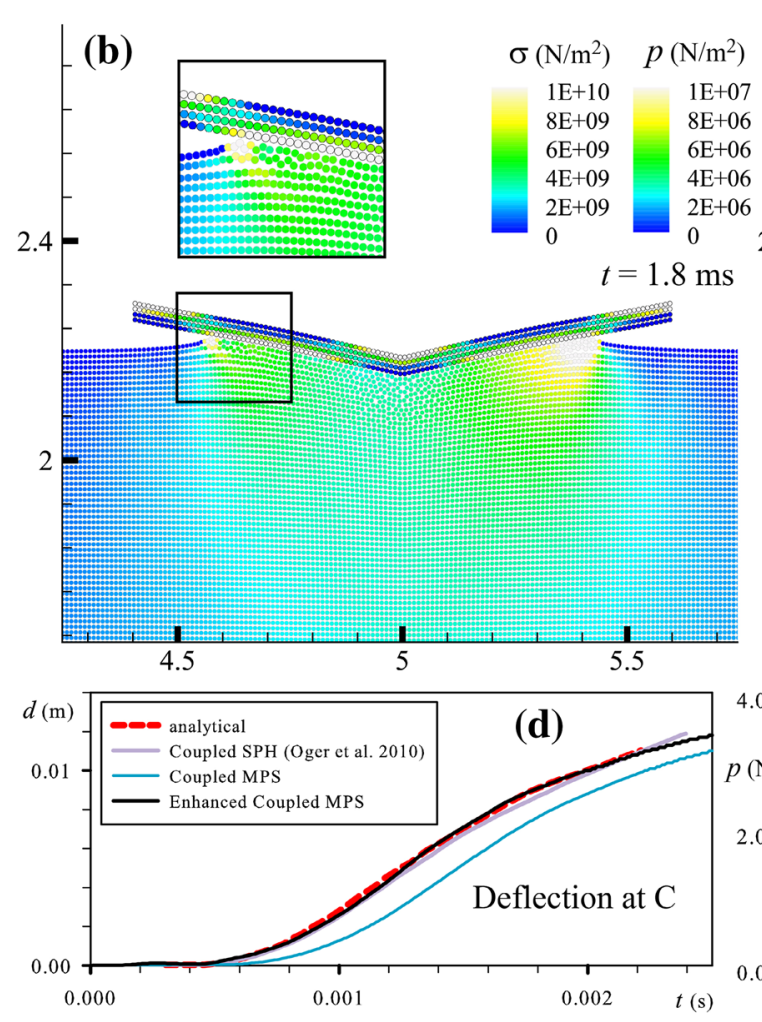

Fig. 9 Simulation results corresponding to an entry of a deformable beam into an undisturbed water (Oger et al. 2010)-schematic sketch of problem (a), snapshots of pressure and stress fields $(\mathbf{b}, \mathbf{c})$, time history

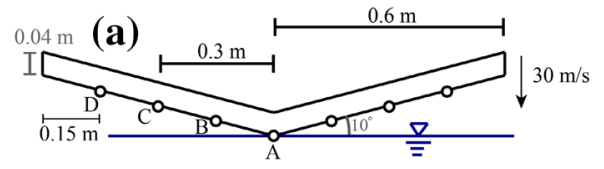

Enhanced Coupled MPS-based FSI Solver

Khayyer et al. (2015b)

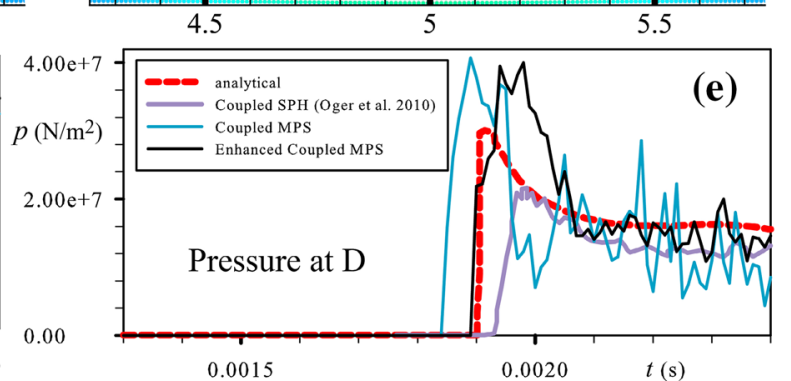

of deflection at measuring point $C(\mathbf{d})$ and pressure at measuring point $D($ e)-improved results by an enhanced coupled MPS-based FSI solver

allel computers, especially on GPUs (graphics processing unit), is definitely among the recent breakthroughs that widens the applicability of these methods. In this context, Hérault et al. (2010) and Crespo et al. (2011) were among the pioneers of implementing SPH on GPUs. They showed that remarkable speedups of up to two orders of magnitude could be achieved by using a single GPU-card in place of a single-core CPU for simulations dealing with more than one million particles. Oger et al. (2016) highlighted various key points corresponding to massive parallelization of explicit particle methods on distributed memory. Mokos et al. (2015) presented a GPU-based implementation of an explicit multiphase SPH code.

In the context of projection-based particle methods, Hori et al. (2011) presented a GPU-based implementation of MPS method. In this case, the speedup was limited to only one order of magnitude, mainly due to iterative solution process of Poisson pressure equation (PPE). The same order of speedup is achieved in other GPU-based implementations of projection-based particle methods, including MPS (e.g. Kakuda et al. 2013) and ISPH (Qiu 2014).

\subsection{Other applications}

An interesting application of particle methods is related to simulation of flow-induced scouring by coupling the fluid solver with an appropriate soil model. By considering the following momentum equations for fluid (Eq. 20) and soil (Eq. 21) as well as a proper stress-strain relationship (Eq. 22; Bui et al. 2008) together with advection-diffusion equation for the suspended sediment (Eq. 23), Ikari et al. (2015a) conduced a study on scouring due to a submerged vertical jet with a sub-particle-scale suspended sediment load model.

$\gamma \rho_{l} \frac{\mathrm{D} \boldsymbol{u}_{l}}{\mathrm{D} t}=-\gamma \nabla p_{l}+\left(\mu+\rho_{l} v_{t}\right) \nabla^{2} \boldsymbol{u}_{l}+\gamma \rho_{l} \boldsymbol{g}+\frac{\gamma^{2} \rho_{l} g}{k_{c}}\left(\boldsymbol{u}_{s}-\boldsymbol{u}_{l}\right)$ 

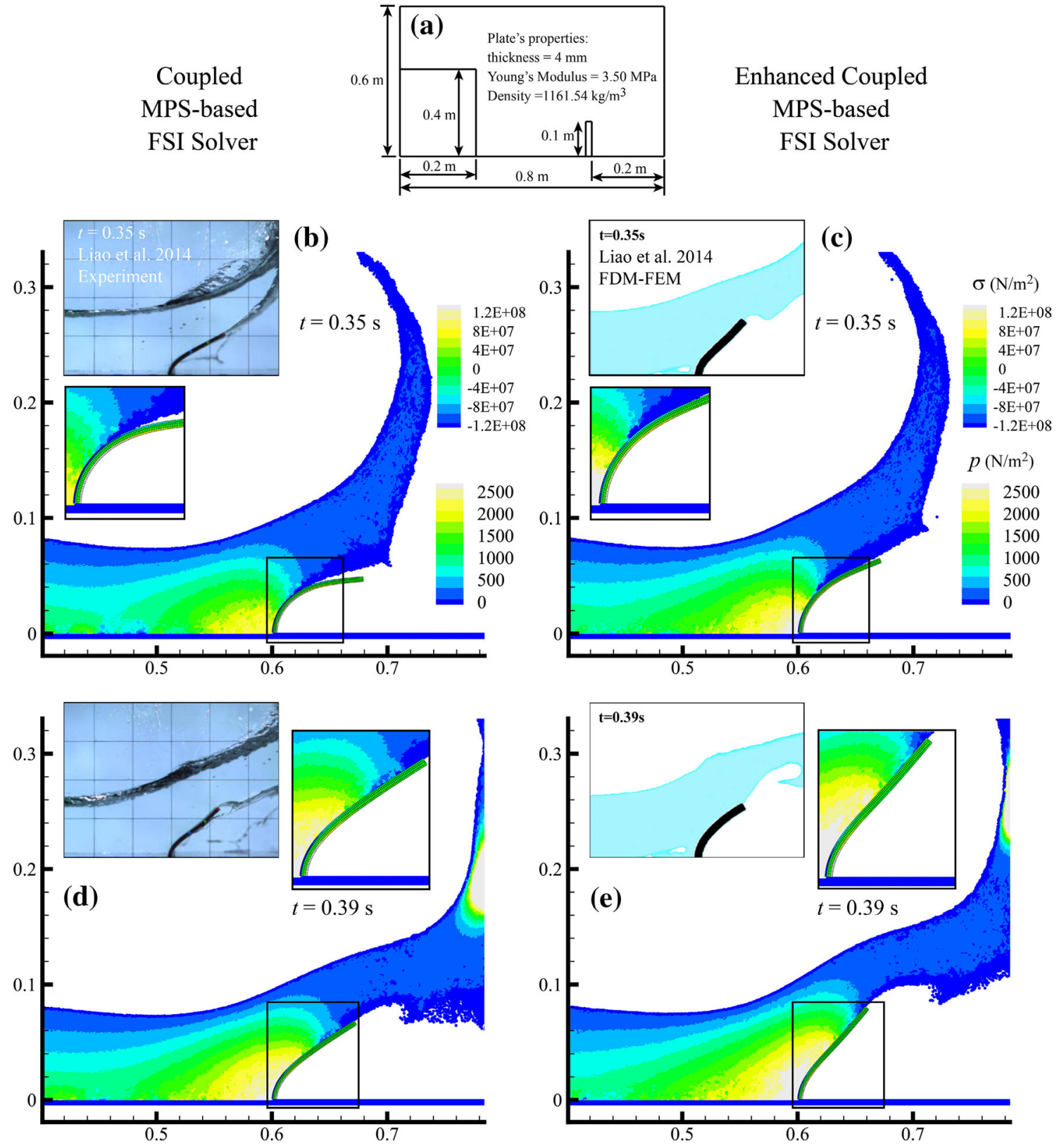

Fig. 10 Simulation results illustrating a dam break with an elastic plate corresponding to the experiment by Liao et al. (2014, 2015)-a schematic sketch of calculation domain (a), results by MPS FSI solver (b, d) and its enhanced version (c, e)

$$
\begin{aligned}
(1-\gamma) \rho_{s} \frac{\mathrm{D} \boldsymbol{u}_{s}}{\mathrm{D} t}= & -\nabla \cdot \boldsymbol{\sigma}-(1-\gamma) \nabla p_{l}+(1-\gamma) \rho_{s} \boldsymbol{g} \\
& +\frac{\gamma^{2} \rho_{l} g}{k_{c}}\left(\boldsymbol{u}_{l}-\boldsymbol{u}_{s}\right) \\
\dot{\boldsymbol{\sigma}}=\dot{\boldsymbol{\omega}} \boldsymbol{\sigma}-\boldsymbol{\sigma} \dot{\boldsymbol{\omega}}+ & \lambda_{e} \operatorname{tr}(\dot{\boldsymbol{\varepsilon}}) \boldsymbol{I}+2 \mu_{e} \dot{\boldsymbol{\varepsilon}}-\dot{\boldsymbol{\sigma}}_{p} \\
\frac{\mathrm{DC}}{\mathrm{D} t}+\boldsymbol{w}_{s} \cdot \nabla C= & \frac{v_{t}}{\sigma_{c}} \nabla^{2} C+Q,
\end{aligned}
$$

where $\gamma$ is the porosity, $\rho$ is the density, $\boldsymbol{u}$ is the velocity, $p$ is the pressure, $\mu$ is the dynamic viscosity coefficient, $v_{t}$ is the kinematic eddy viscosity coefficient, $g, g$ are the gravitational acceleration in absolute and vector forms, $k_{c}$ is the permeability, $\sigma$ is the stress, $\dot{\sigma}$ is the stress rate, $\dot{\omega}$ is the spin tensor, $\lambda_{e}, \mu_{e}$ are the Lamé parameters, $\boldsymbol{I}$ is the unit tensor, $\dot{\varepsilon}$ is the strain rate, $\dot{\sigma}_{p}$ is the stress rate due to plastic strain, $C$ is the concentration, $\boldsymbol{w}_{s}$ is the settling velocity, $\sigma_{c}$ is the Schmidt number and $Q$ is the balance of suspended sediment from sand bed.

Figure 11a shows a set of snapshots corresponding to the conducted simulation, illustrating the jet-induced scouring as well as time evolution of distribution of sub-particle- 

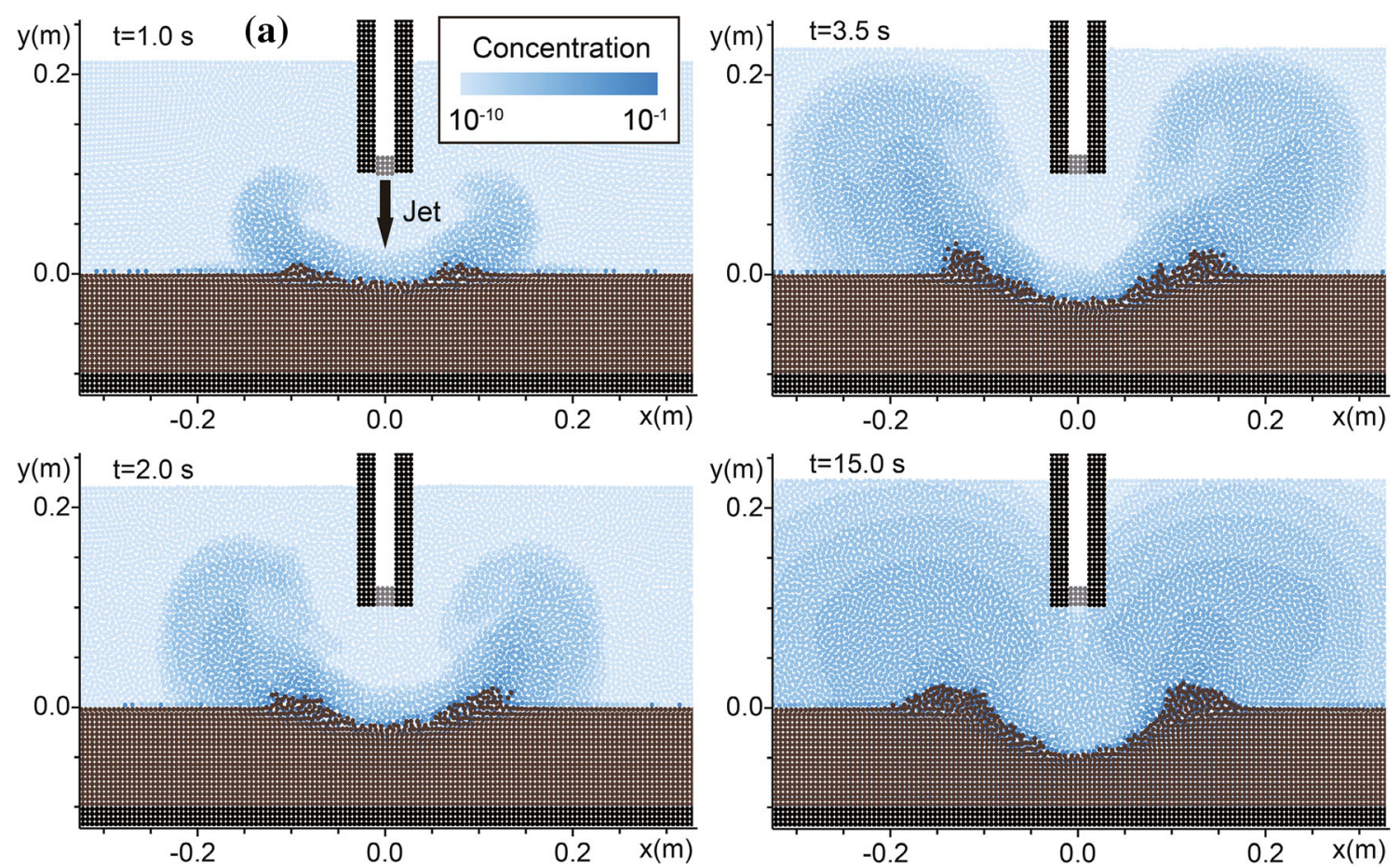

(b)
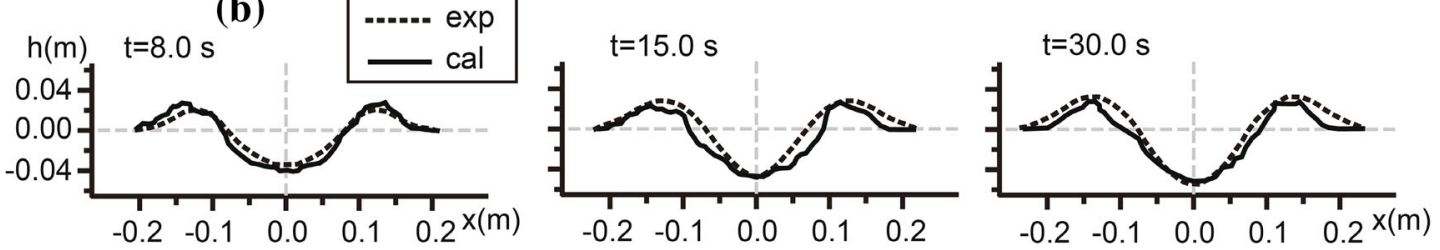

Fig. 11 Simulation results of scouring due to a submerged vertical jet with a sub-particle-scale suspended sediment load model-a snapshots of fluid and soil particles together with suspended sediment concen-

scale suspended sediment. Figure $11 \mathrm{~b}$ presents a quantitative comparison of scouring pattern with the corresponding experiment (Akashi and Saito (1980)), illustrating an almost acceptable agreement, especially in terms of the maximum scour depth. For the scouring simulation presented in Fig. 11, the depth of soil region is $0.1 \mathrm{~m}$ and the water depth above the initial soil-water interface is $0.2 \mathrm{~m}$. The inflow boundary corresponding to the vertical submerged jet has a width of $0.02 \mathrm{~m}$, placed $0.1 \mathrm{~m}$ above the initial soil-water interface. The vertical downward jet has a constant speed of $0.74 \mathrm{~m} / \mathrm{s}$. The mass median diameter (D50) of soil is considered to be $8.4 \mathrm{E}-4$, the same as that in the corresponding experiment (Akashi and Saito 1980). Both fluid and soil particles, i.e. computational spatial resolutions, are considered to be $5 \mathrm{~mm}$ in diameter.

There are also other studies that simply couple the incompressible SPH with simple soil erosion models to estimate the erosion rate and its associated scouring (e.g. Manenti et al. 2012; Ran et al. 2015). A SPH-based two-phase flow model was also presented by Zanganeh et al. (2012) to predict scour- tration, b quantitative comparison of bed profile with corresponding experiment (Akashi and Saito 1980)

ing below marine pipelines. In their study, the soft contact approach of Cundall and Strack (1979) was considered for calculation of inter-particle forces for the granular sediment particles.

In the context of weakly compressible SPH, Ulrich et al. (2013) presented a set of interesting multi-physics marineengineering-related SPH simulations including full-scale applications involving floating-body/water/soil interactions (e.g. installation process of a gravity foundation for offshore wind turbines).

In addition to scouring and suspended-sediment-transport applications, other interesting applications of particle methods in ocean engineering correspond to waves interacting with and flowing through porous structures (e.g. Shao 2010; Akbari and Montazeri Namin 2013), rigid bodies driven by flows (e.g. Tofighi et al. 2015), floating body dynamics (e.g. Shao and Gotoh 2004; Sueyoshi et al. 2008) and nonNewtonian free-surface flows (e.g. Xenakis et al. (2015)).

In general, particle methods, including projection-based ones, are well-suited for multi-scale, multi-physics appli- 
cations, as highlighted by Violeau and Rogers (2016) as well as Liu and Liu (2016). In this regard, coupling of Lagrangian particle methods with either classical Eulerian solvers (e.g. finite volume method or finite element method) or other Lagrangian methods (e.g. Discrete Element Method) has gained interest for efficient and reliable simulations by considering the intrinsic characteristics of each method. Examples include hybrid SPH-FVM (e.g. Marrone et al. 2016) or MPS-FVM (e.g. Liu et al. 2005), as well as SPHFEM (e.g. Chuzel-Marmot et al. 2011), ISPH-FEM (e.g. Asai et al. 2011) or MPS-FEM (e.g. Hashimoto and Le Touzé 2014; Mitsume et al. 2014a, b).

Particle methods have also been coupled with other Lagrangian methods such as DEM for multi-scale simulations of physical phenomena. Examples include hybrid MPS-DEM solvers for simulations of solid-liquid flows (e.g. debris avalanche analysis by Toyoshi et al. 2011) or fluid flow-rigid solid interactions (e.g. wave-armor block interactions in front of a caisson breakwater by Gotoh et al. 2009). In a recent interesting work, Canelas et al. (2016) integrated the advanced contact mechanics theories with $\mathrm{SPH}$ and presented a so-called SPH-DCDEM (Distributed Contact Discrete Element Method) for resolved, accurate simulations of fluid-solid phases.

\section{Future perspectives}

Despite the significant advancements achieved, rigorous and careful researches should be conducted to further enhance the reliability and accuracy of particle methods for practical engineering purposes including those corresponding to ocean engineering.

During the past decade, the stability and accuracy of particle methods, including projection-based ones, have been substantially enhanced. However, as for stability, the currently developed particle methods apply some sort of stabilization schemes such as the particle shifting (Lind et al. 2012) or dynamic stabilization (Tsuruta et al. 2013) ones, in order to guarantee the methods' stability for a wide range of calculations. The probable adverse effects of such schemes in terms of conservation and convergence must be rigorously studied. The stability of particle methods is also preferred to be enhanced by refinement of differential operator models, such as gradient and Laplacian operators, corresponding to the terms that directly appear in the considered governing equations, or through applications of higher-order accurate numerical solution processes (e.g. higher-order projection methods, for instance).

As for accuracy, in spite of significant improvements, the problem of unphysical pressure fluctuations remain to be not fully resolved. Further enhancements of accuracy are expected to be achieved thanks to the profound and meticulous studies that are being conducted in this field.

As for boundary conditions and as it was stated in this paper, several important developments have been made during the past couple of years. The ongoing and future researches will further target this important aspect with rigorous studies on consistency, conservation and convergence of implemented boundary conditions. In particular, development of more accurate, consistent free-surface and inflow-outflow boundary conditions will further enhance the reliability of particle methods for ocean engineering applications.

As for enhancement of energy conservation properties of projection-based particle methods, special focus should be given to revisit/derive the formulations with respect to an energy-based framework. In this regard, compatibility of differential operator models is a key issue, as highlighted in Sect. 2.3 .

As for multiphase flow simulations, especially those characterized by large density ratios, the currently developed particle methods consider some numerical treatments, such as stabilizers or smoothing schemes. Further reliable multiphase particle methods are expected to be proposed as the stability and accuracy of these methods progress.

As for fluid-structure interactions corresponding to deformable structures, further advancements are expected by improvements in both flow field as well as incorporated mathematical/numerical models for the structure.

An important research category for further enhancement of the reliability of particle methods for ocean engineering applications corresponds to turbulence modeling. Up to now, several studies have incorporated different types of turbulence models in the context of both explicit particle methods (e.g. Issa et al. 2010; De Padova et al. 2013; Mayrhofer 2014) and the semi-implicit projection-based ones (e.g. Shao and Lo 2003; Gotoh and Sakai 2006; Leroy et al. 2015a). Researches on proper modeling of turbulence by either timeaveraged (e.g. Violeau and Issa 2007) or spatially averaged (e.g. sub-particle-scale; Gotoh et al. 2001) turbulence models are continuously advancing (e.g. Mayrhofer et al. 2015).

As for future applications, couplings of proper soil (e.g. Ikari and Gotoh 2016) and structure models with projection-based ISPH or MPS methods potentially result in enhanced multi-scale, multi-physics simulations including those related to ocean engineering (e.g. submarine debris flow impact on pipelines). Further advanced multiscale and multi-physics applications of particle methods are expected to be achieved with forthcoming theoretical and computational enhancements. In particular, enhancements of stability, accuracy and conservation properties of particle methods along with advancements made in highperformance computing as well as developments of accurate variable resolution schemes (e.g. Vacondio et al. 2016) will 
enable particle methods to serve as advanced, reliable and efficient computational methods.

In all cases, it is important to keep the developed numerical methods free of any numerical term with constants that require calibration. This important issue is also highlighted in an excellent review paper by Violeau and Rogers (2016). Indeed, prior to any practical application, rigorous and meticulous verification of particle-based codes must be conducted by consideration of appropriate benchmark tests with analytical solutions in terms of reproduced velocity, pressure together with comprehensive investigations on conservation and convergence properties.

\section{Concluding remarks}

Current achievements corresponding to development of particle methods with applications in ocean engineering are discussed, with special focus on a distinct category of these methods, namely, projection-based particle methods, including both MPS and ISPH methods. Latest advancements corresponding to enhancements of stability, accuracy, energy conservation, boundary conditions and improved simulations of multiphase flows and fluid-structure interactions are reviewed. The future perspectives for further development of these methods for more reliable applications in engineering fields, including ocean engineering, are also highlighted.

Acknowledgments The authors would like to express their gratitude to Dr. Hiroyuki Ikari at Department of Civil and Earth Resources Engineering, Kyoto University, as well as Dr. Naoki Tsuruta at Japan's Port and Airport Research Institute for their contributions in conducting some of the simulations that are presented in this paper.

\section{References}

Adami S, Hu XY, Adams NA (2010) A new surface-tension formulation for multi-phase sph using a reproducing divergence approximation. J Comput Phys 229:5011-5021

Adami S, Hu XY, Adams NA (2012) A generalized wall boundary condition for smoothed particle hydrodynamics. J Comput Phys 231(21):7057-7075

Adami S, Hu XY, Adams NA (2013) A transport-velocity formulation for smoothed particle hydrodynamics. J Comput Phys 241:292307

Akashi N, Saito T (1980) Studies on the scour from submerged impinged jet. Proc Jpn Soc Civ Eng 298:53-62 (in Japanese)

Akbari H, Montazeri Namin M (2013) Moving particle method for modeling wave interaction with porous structures. Coast Eng 74:59-73

Alam A, Kai H, Suzuki K (2007) Two-dimensional numerical simulation of water splash phenomena with and without surface tension. J Mar Sci Technol 12:59-71

Altomare C, Crespo AJC, Domínguez JM, Gómez-Gesteira M, Suzuki T, Verwaest T (2015) Applicability of smoothed particle hydrodynamics for estimation of sea wave impact on coastal structures. Coast Eng 96:1-12
Alvarado-Rodriguez CE, Barreiro A, Dominguez JM, Crespo AJC, Gomez Gesteira M, Klapp J (2015) Simulation of dispersion in a porous media with multiphase smoothed particle hydrodynamics. In: Proceedings of 10th International SPHERIC workshop, Parma, 9-14, 2015

Amanifard N, Hesan M, Rahbar B (2011) An SPH approach for fluid-hypoelastic structure interaction with free surfaces. In: Proceedings of the world congress on Engineering, Vol III, July 6-8, 2011, London

Antoci C, Gallati M, Sibilla S (2007) Numerical simulation of fluidstructure interaction by SPH. Comput Struct 85(11):879-890

Antuono M, Colagrossi A, Marrone S (2012) Numerical diffusive terms in weakly-compressible SPH schemes. Comput Phys Commun 183:2570-2580

Asai M, Aly AM, Sonoda Y (2011) ISPH-FEM coupling simulator for the FSI problems. In: Proceedings of 6th International SPHERIC Workshop, Hamburg University of Technology (TUHH), 8-9 June 2011, pp 201-208. ISBN: 978-3-89220-658-3

Ataie-Ashtiani B, Shobeyri G, Farhadi L (2008) Modified incompressible SPH method for simulating free surface problems. Fluid Dyn Res 40(9):637-661

Balsara DS (1995) Von Neumann stability analysis of smoothed particle hydrodynamics - suggestions for optimal algorithms. J Comput Phys 121:357-372

Basa M, Quinlan JN, Lastiwka M (2009) Robustness and accuracy of SPH formulations for viscous flow. Int J Numer Methods Fluids 60(10):1127-1148

Beissel S, Belytschko T (1996) Nodal integration of the element-free Galerkin method. Comput Methods Appl Mech Eng 139:49-74

Belytschko T, Guo Y, Liu WK, Xiao SP (2000) A unified stability analysis of meshless particle methods. Int J Numer Methods Eng 48(9):1359-1400

Belytschko T, Xiao SP (2002) Stability analysis of particle methods with corrected derivatives. Comput Math Appl 43(3):329-350

Bonet J, Lok TS (1999) Variational and momentum preservation aspects of smooth particle hydrodynamic formulation. Comput Methods Appl Mech Eng 180:97-115

Brackbill JU, Kothe DB, Zemach C (1992) A continuum method for modeling surface tension. J Comput Phys 100:335-354

Bui H, Fukagawa R, Sako K, Ohno S (2008) Lagrangian meshfree particle method (SPH) for large deformation and failure flows of geomaterial using elastic-plastic soil constitutive model. Int J Numer Anal Methods Geomech 32:1537-1570

Canelas RB, Crespo AJC, Domínguez JM, Ferreira RML, GómezGesteira M (2016) SPH-DCDEM model for arbitrary geometries in free surface solid-fluid flows. Comput Phys Commun 202:131140

Chen JK, Beraun JE, Jih CJ (1999) An improvement for tensile instability in smoothed particle hydrodynamics. Comput Mech 23:279-287

Chorin AJ (1968) Numerical solution of the Navier-Stokes equations. Math Comput 22:745-762

Chuzel-Marmot Y, Ortiz R, Combescure A (2011) Three dimensional SPH-FEM gluing for simulation of fast impacts on concrete slabs. Comput Struct 89:2484-2494

Colagrossi A, Marrone S, Bouscasse B, Broglia R (2015) Numerical Simulations of the Flow Past Surface-Piercing Objects. International Journal of Offshore and Polar Engineering 25(1):13-18

Colagrossi A, Antuono M, Souto-Iglesias A, Le Touzé D (2011) Theoretical analysis and numerical verification of the consistency of viscous smoothed-particle-hydrodynamics formulations in simulating free-surface flows. Phys Rev E, 84(2):026705. doi:10.1103/ PhysRevE.84.026705

Colagrossi A, Landrini M (2003) Numerical simulation of interfacial flows by smoothed particle hydrodynamics. J Comput Phys 191(2):448-475 
Crespo AC, Dominguez JM, Barreiro A, Gómez-Gesteira M, Rogers BD (2011) GPUs, a new tool of acceleration in CFD: efficiency and reliability on smoothed particle hydrodynamics methods. PLoS One 6:e20685

Cummins SJ, Rudman M (1999) An SPH projection method. J Comput Phys 152:584-607

Cundall PA, Strack ODL (1979) A discrete numerical model for granular assemblies. Geotechnique 29(1):47-65

Dalrymple RA, Rogers BD (2006) Numerical modeling of water waves with the SPH method. Coast Eng 53(2):141-147

De Padova D, Mossa M, Sibilla S, Torti E (2013) 3D SPH modelling of hydraulic jump in a very large channel. J Hydraul Res 51(2):158173

Dehnen W, Aly H (2012) Improving convergence in smoothed particle hydrodynamics simulations without pairing instability. Mon Not R Astron Soc 425(2): 1068-1082

Delorme L, Colagrossi A, Souto-Iglesias A, Zamora-Rodriguez R, Botia-Vera E (2009) A set of canonical problems in sloshing, part I: pressure field in forced roll-comparison between experimental results and SPH. Ocean Eng 36(2):168-178

Di Monaco A, Manenti S, Gallati M, Sibilla S, Agante G, Guandalini R (2011) SPH modeling of solid boundaries through a semi-analytic approach. Eng Appl Comput Fluid Mech 5(1):1-15

Dilts GA (1999) Moving least squares hydrodynamics: consistency and stability. Int J Numer Methods Eng 44(8):1115-1155

Duan G, Koshizuka S, Chen B (2015) A contoured continuum surface force model for particle methods. J Comput Phys 298:280-304

Dyka CT, Randles PW, Ingel RP (1997) Stress points for tension instability in SPH. Int J Numer Methods Eng 40(13):2325-2341

Fang J, Parriaux A, Rentschler M, Ancey C (2009) Improved SPH methods for simulating free surface flows of viscous fluids. Appl Numer Math 59(2):251-271

Farahani RJ, Dalrymple RA, Hérault A, Bilotta G (2014) Threedimensional SPH modeling of a bar/rip channel system. J Waterw Port Coast Ocean Eng 140(1):82-99

Farahani RJ, Dalrymple RA (2014) Three-dimensional reversed horseshoe vortex structures under broken solitary waves. Coast Eng 91:261-279

Fatehi R, Manzari MT (2011a) Error estimation in smoothed particle hydrodynamics and a new scheme for second derivatives. Comput Math Appl 61(2):482-498

Fatehi R, Manzari MT (2011b) A remedy for numerical oscillations in weakly compressible smoothed particle hydrodynamics. Int $\mathrm{J}$ Numer Methods Fluids 67:1100-1114

Ferrand M, Laurence DR, Rogers BD, Violeau D, Kassiotis C (2013) Unified semi analytical wall boundary conditions for inviscid, laminar or turbulent flows in the meshless SPH method. Int J Numer Methods Fluids 71(4):446-472

Fries TP, Belytschko T (2008) Convergence and stabilization of stresspoint integration in mesh-free and particle methods. Int J Numer Methods Eng 74(7):1067-1087

Fu L, Jin YC (2015) Investigation of non-deformable and deformable landslides using meshfree method. Ocean Eng 109:192-206

Gao R, Ren B, Wang GY, Wang YX (2012) Numerical modelling of regular wave slamming on subface of open-piled structures with the corrected SPH method. Appl Ocean Res 34:173-186

Gingold RA, Monaghan JJ (1977) Smoothed particle hydrodynamics: theory and application to non-spherical stars. Mon Not R Astron Soc 181:375-389

Gotoh H, Shibahara T, Sakai T (2001) Sub-particle-scale turbulence model for the mps method-lagrangian flow model for hydraulic engineering. Comput Fluid Dyn J 9(4):339-347

Gotoh H, Ikari H, Memita T, Sakai T (2005) Lagrangian particle method for simulation of wave overtopping on a vertical seawall. Coast Eng J 47(2\&3):157-181
Gotoh H (2009) Lagrangian particle method as advanced technology for numerical wave flume. Int J Offshore Polar Eng 19(3):161-167

Gotoh H, Khayyer A, Ikari H, Arikawa T, Shimosako K (2014) On enhancement of incompressible SPH method for simulation of violent sloshing flows. Appl Ocean Res 46:104-105

Gotoh H, Fredsøe J (2000) Lagrangian two-phase flow model of the settling behavior of fine sediment dumped into water. In: Proc. ICCE, Sydney, pp 3906-3919

Gotoh H, Ikari H, Sakai T (2004) Simulation of falling water by lagrangian particle method. In: Advances in hydro-science and engineering, vol VI, Proceedings of 6th ICHE, Brisbane

Gotoh H, Ikari H, Yasuoka T (2009) Simulation of armor blocks in front of caisson breakwater by DEM-MPS hybrid model. In: Proceedings of 19th international offshore and polar engineering conference, Osaka, pp 365-370

Gotoh H, Okayasu A, Watanabe Y (2013) Computational wave dynamics. World Scientific Publishing Co, p 234. ISBN: 978-981-444970-0

Gotoh H, Sakai T (1999) Lagrangian simulation of breaking waves using particle method. Coast Eng J 41:303-326

Gotoh H, Sakai T (2006) Key issues in the particle method for computation of wave breaking. Coast Eng 53(2):171-179

Gray JP, Monaghan JJ, Swift RP (2001) SPH elastic dynamics. Comput Methods Appl Mech Eng 190:6641-6662

Grenier N, Antuono M, Colagrossi A, Le Touzé D, Alessandrini B (2009) An Hamiltonian interface SPH formulation for multi-fluid and free surface flows. J Comput Phys 228:8380-8393

Guenther C, Hicks DL, Swegle JW, (1994) Conservative smoothing versus artificial viscosity. No. SAND-94-1853. Sandia National Labs., Albuquerque

Hashimoto H, Le Touzé D (2014) Coupled MPS-FEM model for violent flows-structures interaction. In: Proceedings of the 29th workshop on water waves and floating bodies, Osaka

Hérault A, Billotta G, Dalrymple RA (2010) SPH on GPU with CUDA. J Hydraul Res 48:74-79

Hicks DL, Liebrock LM (2004) Conservative smoothing with B-splines stabilizes SPH material dynamics in both tension and compression. Appl Math Comput 150:213-234

Hopkins PF (2015) A new class of accurate, mesh-free hydrodynamic simulation methods. Mon Not R Astrono Soc 450(1):53-110

Hori C, Gotoh H, Ikari H, Khayyer A (2011) GPU-acceleration for moving particle semi-implicit method. Comput Fluids 51(1):174183

Hosseini SM, Feng JJ (2011) Pressure boundary conditions for computing incompressible flows with SPH. J Comput Phys 230:74737487

Hu XY, Adams NA (2006) A multi-phase SPH method for macroscopic and mesoscopic flows. J Comput Phys 213:844-861

Hu XY, Adams NA (2007) An incompressible multi-phase SPH method. J Comput Phys 227:264-278

Hughes JP, Graham DI (2010) Comparison of incompressible and weakly-compressible SPH models for free-surface water flows. J Hydraul Res 48:105-117

Hwang SC, Khayyer A, Gotoh H, Park JC (2014) Development of a fully Lagrangian MPS-based coupled method for simulation of fluid-structure interaction problems. J Fluids Struct 50:497-511

Ichikawa H, Labrosse S (2010) Smooth particle approach for surface tension calculation in moving particle semi-implicit method. Fluid Dyn Res 42:035503

Ikari H, Khayyer A, Gotoh H (2015b) Corrected higher order Laplacian for enhancement of pressure calculation by projection-based particle methods with applications in ocean engineering. J Ocean Eng Mar Energy 1:361-376

Ikari H, Gotoh H (2016) SPH-based simulation of granular collapse on an inclined bed. Mech Res Commun 73:12-18 
Ikari H, Gotoh H, Sakai T (2004) Simulation of wave breaking by the particle method with liquid-gas two-phase flow model. Annu J Coast Eng, pp 111-115 (in Japanese)

Ikari H, Gotoh H, Tanbo T, Ejiri T (2015a) MPS-based simulation of scouring due to submerged vertical jet with sub-particle-scale suspended sediment model. J Jpn Soc Civ Eng Ser. B2 (Coastal Engineering) 71:19-24

Inutsuka S (1994) Godunov-type SPH. J Ital Astron Soc 65:1027-1031

Inutsuka S (2002) Reformulation of smoothed particle hydrodynamics with Riemann solver. J Comput Phys 179:238-267

Ishii E, Kohira H (2009) Simulation of lubricant behavior by using the particle method. IEEE Trans Magn 45(11):5044-5049

Ishii E, Sugii T (2011) Development of surface tension model using inter-particle force in particle method based on continuum dynamics. In: International mechanical engineering congress and exposition IMECE, pp 11-17

Issa R, Violeau D, Lee ES, Flament H (2010) Modelling nonlinear water waves with RANS and LES SPH models. In: Ma QW (ed) Advances in numerical simulation of nonlinear water waves, vol 11. World Scientific Publishing Co, ch. 14

Jiang T, Ouyang J, Ren JL, Yang BX, Xu XY (2012) A mixed corrected symmetric SPH (MC-SSPH) method for computational dynamic problems. Comput Phys Commun 183:50-62

Johnson GR, Stryk RA, Beissel SR (1996) SPH for high velocity impact computations. Comput Methods Appl Mech Eng 139:347-373

Kakuda K, Nagashima T, Hayashi Y, Obara S, Toyotani J, Miura S, Matsuda S (2013) Three-dimensional fluid flow simulations using GPU-based particle method. Comput Model Eng Sci 93(5):363376

Kassiotis C, Violeau D, Ferrand M (2013) Semi-analytical conditions for open boundaries in smoothed particle hydrodynamics. In: Proceedings of 8th international SPHERIC workshop, paper 1-4

Khayyer A, Gotoh H, Shao SD (2008) Corrected incompressible SPH method for accurate water-surface tracking in breaking waves. Coast Eng 55(3):236-250

Khayyer A, Gotoh H, Shao SD (2009) Enhanced predictions of wave impact pressure by improved incompressible SPH methods. Appl Ocean Res 31(2):111-131

Khayyer A, Gotoh H, Tsuruta N (2014) A new surface tension for particle methods with enhanced splash computation. J Jpn Soc Civ Eng Ser B2 (Coastal Engineering) 70(2):26-30

Khayyer A, Gotoh H (2008) Development of CMPS method for accurate water-surface tracking in breaking waves. Coast Eng J 50(2):179_ 207

Khayyer A, Gotoh H (2009a) Modified moving particle semi-implicit methods for the prediction of 2D wave impact pressure. Coast Eng $56: 419-440$

Khayyer A, Gotoh H (2009b) Wave impact pressure calculations by improved SPH methods. Int J Offshore Polar Eng 19(4):300-307

Khayyer A, Gotoh H (2010a) On particle-based simulation of a dam break over a wet bed. J Hydraul Res IAHR 48(2):238-249

Khayyer A, Gotoh H (2010b) A higher order laplacian model for enhancement and stabilization of pressure calculation by the MPS method. Appl Ocean Res 32(1):124-131

Khayyer A, Gotoh H (2011) Enhancement of stability and accuracy of the moving particle semi-implicit method. J Comput Phys 230:3093-3118

Khayyer A, Gotoh H (2012) A 3D higher order laplacian model for enhancement and stabilization of pressure calculation in 3D MPSbased simulations. Appl Ocean Res 37:120-126

Khayyer A, Gotoh H (2013) Enhancement of performance and stability of MPS meshfree particle method for multiphase flows characterized by high density ratios. J Comput Phys 242:211-233

Khayyer A, Gotoh H (2016) A multiphase compressible-incompressible particle method for water slamming. Int J Offshore Polar Eng 26(1):20-25
Khayyer A, Gotoh H, Park JC, Hwang SC, Koga T (2015b) An enhanced fully Lagrangian coupled MPS-based solver for fluid-structure interactions. J JSCE (Coastal Eng.) 71:883-888. ISSN:1884-2399

Khayyer A, Gotoh H, Shimizu Y, Gotoh K (2015a) On enhancement of energy conservation properties of ISPH and MPS methods. In: Proceedings of 10th international SPHERIC workshop, Parma, pp 139-146

Khorasanizade S, Sousa JMM (2016) An innovative open boundary treatment for incompressible SPH. Int J Numer Methods Fluids 80:161-180

Kondo M, Suzuki Y, Koshizuka S (2010) Suppressing local particle oscillations in the Hamiltonian particle method for elasticity. Int J Numer Methods Eng 81(12):1514-1528

Kondo M, Koshizuka S (2011) Improvement of stability in moving particle semi-implicit method. Int J Numer Methods Fluids 65:638-654

Kondo M, Koshizuka S, Suzuki K. Takimoto M (2007) Surface tension model using inter-particle force in particle method. Proc FEDSM 2007:93-98. doi:10.1115/FEDSM2007-37215

Koshizuka S, Nobe A, Oka Y (1998) Numerical analysis of breaking waves using the moving particle semi-implicit method. Int J Numer Methods Fluid 26:751-769

Koshizuka S (2011) Current achievements and future perspectives on particle simulation technologies for fluid dynamics and heat transfer. Journal of Nuclear Science and Technology 48(2):155-168

Koshizuka S, Oka Y (1996) Moving particle semi-implicit method for fragmentation of incompressible fluid. Nucl Sci Eng 123:421-434

Lastiwka M, Basa M, Quinlan NJ (2009) Permeable and non-reflecting boundary conditions in SPH. Int J Numer Methods Fluids 61:709724

Le Touzé D, Marsh A, Oger G, Guilcher PM, Khaddaj-Mallat C, Alessandrini B, Ferrant P (2010) SPH simulation of green water and ship flooding scenarios. J Hydrodyn Ser B 22(5):231-236

Le Touzé D, Colagrossi A, Colicchio G, Greco M (2013) A critical investigation of smoothed particle hydrodynamics applied to problems with free surfaces. Int J Numer Methods Fluids 73:660-691

Lee CJK, Noguchi H, Koshizuka S (2007) Fluid-shell structure interaction analysis by coupled particle and finite element method. Comput Struct 85:668-697

Lee ES, Moulinec C, Xu R, Violeau D, Laurence D, Stansby P (2008) Comparisons of weakly compressible and truly incompressible algorithms for the SPH mesh free particle method. J Comput Phys 227(18):8417-8436

Lee BH, Park JC, Kim MH, Hwang SC (2011) Step-by-step improvement of MPS method in simulating violent free-surface motions and impact-loads. Comput Methods Appl Mech Eng 200(912):1113-1125

Lee BH, Jeong S, Hwang SC, Park JC, Kim MH (2013) A particle simulation of 2-D vessel motions interacting with liquid-sloshing cargo. Comput Model Eng Sci 91(1):43-63

Leroy A, Violeau D, Ferrand M, Kassiotis C (2014) Unified semianalytical wall boundary conditions applied to 2-D incompressible SPH. J Comput Phys 261:106-129

Leroy A, Violeau D, Ferrand M, Joly A (2015a) Buoyancy modelling with incompressible SPH for laminar and turbulent flows. Int J Numer Methods Fluids 78(8):455-474

Leroy A, Violeau D, Joly A, Fratter L, Ferrand M (2015b) Open boundary conditions for ISPH with the unified semi-analytical boundary conditions. In: Proceedings of 10th international SPHERIC workshop, pp 316-324

Liao K, Hu C, Sueyoshi M (2015) Free surface flow impacting on an elastic structure: experiment versus numerical simulation. Appl Ocean Res 50:192-208

Liao K, Hu C, Sueyoshi M (2014) Numerical simulation of free surface flow impacting on an elastic plate. In: Proceedings of 29th Intl workshop on water wave and floating bodies, Osaka, March 30April 2, 2014 
Libersky LD, Petschek AG, Carney TC, Hipp JR, Allahdadi FA (1993) High strain Lagrangian hydrodynamics: a three-dimensional SPH code for dynamic material response. J Comput Phys 109(1):67-75

Lind SJ, Xu R, Stansby PK, Rogers BD (2012) Incompressible smoothed particle hydrodynamics for free-surface flows: a generalised diffusion-based algorithm for stability and validations for impulsive flows and propagating waves. J Comput Phys 231(4):1499-1523

Lind SJ, Stansby PK, Rogers BD, Lloyd PM (2015) Numerical predictions of water-air wave slam using incompressible-compressible smoothed particle hydrodynamics. Appl Ocean Res 49:57-71

Lind SJ, Stansby PK, Rogers BD (2016) Incompressible-compressible flows with a transient discontinuous interface using smoothed particle hydrodynamics (SPH). J Comput Phys 309:129-147

Lin MC, Shieh LD (1997) Simultaneous measurements of water impact on a two-dimensional body. Fluid Dyn Res 19:125-148

Liow JL (2001) Splash formation by spherical drops. J Fluid Mech 427:73-105

Liu MB, Liu GR (2016) Particle methods for multi-scale and multiphysics. World Scientific Publishing Co, p 400. ISBN: 978-9814571-69-2

Liu J, Koshizuka S, Oka Y (2005) A hybrid particle-mesh method for viscous, incompressible, multiphase flows. J Comput Phys 202:65-93

Liu X, Xu H, Shao SD, Lin P (2013) An improved incompressible SPH model for simulation of wave-structure interaction. Comput Fluids 71:113-123

Liu X, Lin P, Shao SD (2015) ISPH wave simulation by using an internal wave maker. Coast Eng 95:160-170

Ma ZH, Causon DM, Qian L, Mingham CG, Gu HB, Martinez Ferrer P (2014) A compressible multiphase flow model for violent aerated wave impact problems. Proc R Soc A 470:2172

Macià F, Antuono M, Gonzales LM, Colagrossi A (2011) Theoretical analysis of the no-slip boundary condition enforcement in SPH methods. Prog Theor Phys 125(6):1091-1121

Macià F, González LM, Cercos-Pita JL, Souto-Iglesias A (2012) A boundary integral SPH formulation consistency and applications to ISPH and WCSPH. Prog Theor Phys 128:439-462

Manenti S, Sibilla S, Gallati M, Agate G, Guandalini R (2012) SPH simulation of sediment flushing induced by a rapid water flow. J Hydraul Eng 138(3):272-284

Marrone S, Colagrossi A, Di Mascio A, Le Touzé D (2015) Prediction of energy losses in water impacts using incompressible and weakly compressible models. J Fluids Struct 54:802-822

Marrone S, Di Mascio A, Le Touzé D (2016) Coupling of smoothed particle hydrodynamics with finite volume method for free-surface flows. J Comput Phys 310:161-180

Mayrhofer A (2014) Large Eddy simulation with smoothed particle hydrodynamics: an investigation into wall boundary conditions and turbulent flows. PhD thesis, University of Manchester, Manchester

Mayrhofer A, Rogers BD, Violeau D, Ferrand M (2013) Investigation of wall bounded flows using SPH and the unified semi-analytical wall boundary conditions. Comput Phys Commun 184(11):2515-2527

Mayrhofer A, Laurence D, Rogers BD, Violeau D (2015) DNS and LES of 3-D wall-bounded turbulence using smoothed particle hydrodynamics. Comput Fluids 115:86-97

Ma QW, Zhou JT (2009) MLPG_R method for numerical simulation of 2-D breaking waves. Comput Model Eng Sci 43(3):277-303

Mitsume N, Yoshimura S, Murotani K, Yamada T (2014a) MPS-FEM partitioned coupling approach for fluid-structure interaction with free surface flow. Int J Comput Methods 11(4):135-151

Mitsume N, Yoshimura S, Murotani K, Yamada T (2014b) Improved MPS-FE fluid-structure interaction coupled method with MPS polygon wall boundary model. Comput Model Eng Sci (CMES) 101(4):229-247
Mokos A, Rogers BD, Stansby PK, Domínguez JM (2015) Multi-phase SPH modelling of violent hydrodynamics on GPUs. Comput Phys Commun 196:304-316

Monaghan JJ (1992) Smoothed particle hydrodynamics. Annu Rev Astron Astrophys 30:543-574

Monaghan JJ (1994) Simulating free surface flow with SPH. J Comput Phys 110(2):399-406

Monaghan JJ (1997) SPH and Riemann solvers. J Comput Phys 136(2):298-307

Monaghan JJ (2000) SPH without a tensile instability. J Comput Phys 159(2):290-311

Monaghan JJ (2005) Smoothed particle hydrodynamics. Rep Progr Phys 68(8): 1703

Monaghan JJ, Price DJ (2001) Variational principles for relativistic smoothed particle hydrodynamics. Mon Not R Astron Soc 328:381-392

Monaghan JJ, Rafiee A (2013) A simple SPH algorithm for multi-fluid flow with high density ratios. Int J Numer Methods Fluids 71:537561

Morris JP (1996) Analysis of smoothed particle hydrodynamics with applications. Ph.D. thesis, Monash University, Melbourne

Morris JP, Fox PJ, Zhu Y (1997) Modelling low Reynolds number incompressible flows using SPH. J Comput Phys 136:214-226

Morris JP (2000) Simulating surface tension with smoothed particle hydrodynamics. Int J Numer Methods Fluids 33:333-353

Nair P, Tomar G (2014) An improved free surface modeling for incompressible SPH. Comput Fluids 102:304-314

Natsui S, Soda R, Kon T, Ueda S, Kano J, Inoue R, Ariyama T (2012) Wettability model considering three-phase interfacial energetics in particle method. Mater Trans 53(4):662-670

Ngo-Cong D, Tran CD, Mai-Duy N, Tran-Cong T (2015) Incompressible smoothed particle hydrodynamics-moving IRBFN method for viscous flow problems. Eng Anal Bound Elem 59:172-186

Nomura K, Koshizuka S, Oka Y, Obata H (2001) Numerical analysis of droplet breakup behavior using particle method. J Nucl Sci Technol 38(12):1057-1064

Nugent S, Posch HA (2000) Liquid drops and surface tension with smoothed particle applied mechanics. Phys Rev E 62:4968-4975

Oger G, Doring M, Alessandrini B, Ferrant P (2007) An improved SPH method: towards higher order convergence. J Comput Phys 225(2):1472-1492

Oger G, Guilcher PM, Jacquin E, Brosset L, Deuff JB, Le Touzé D (2010) Simulations of hydro-elastic impacts using a parallel SPH model. Int J Offshore Polar Eng 20(3):181-189

Oger G, Le Touzé D, Guibert D, de Leffe M, Biddiscombe J, Soumagne J, Piccinali JG (2016) On distributed memory MPI-based parallelization of SPH codes in massive HPC context. Comput Phys Commun 200:1-14

Oger G, Marrone S, Le Touzé D (2015) A consistent continuous particle reordering in weakly-compressible SPH through an ALE formalism. In: Proceedings of 10th international SPHERIC workshop, Parma, pp 201-207

Panizzo A, Dalrymple RA (2004) SPH modelling of underwater landslide generated waves. In: Proc. 29th Int. Conf. Coastal Eng., Lisbon, ASCE, pp 1147-1159

Park JI, Park JC, Hwang SC, Heo JK (2014) Two-dimensional particle simulation for behaviours of floating body near quaywall during tsunami. J Ocean Eng Technol 28(1):12-19

Park S, Jeun G (2011a) Calculation of water droplet impingement using the coupled method of rigid body dynamics and the moving particle semi-implicit method. J Mech Sci Technol 25(11):2787-2794

Park S, Jeun G (2011b) Coupling of rigid body dynamics and moving particle semi-implicit method for simulating isothermal multi-phase fluid interactions. Comput Methods Appl Mech Eng 200:130-140 
Qiang HF, Chen FZ, Gao WR (2011) Modified algorithm for surface tension with smoothed particle hydrodynamics and its applications. Comput Model Eng Sci 77:239-262

Qiu LC (2014) OpenCL-based GPU acceleration of ISPH simulation for incompressible flows. Appl Mech Mater 444:380-384

Rabczuk T, Belytschko T, Xiao SP (2004) Stable particle methods based on Lagrangian kernels. Comput Methods Appl Mech Eng 193(1214):1035-1063

Rafiee A, Cummins S, Rudman M, Thiagarajan K (2012) Comparative study on the accuracy and stability of SPH schemes in simulating energetic free-surface flows. Eur J Mech B/Fluids 36:1-16

Rafiee A, Thiagarajan KP (2009) An SPH projection method for simulating fluid-hypoelastic structure interaction. Comput Methods Appl Mech Eng 198:2785-2795

Ran Q, Tong J, Shao SD, Fu X, Xu Y (2015) Incompressible SPH scour model for movable bed dam break flows. Adv Water Resour 82:39-50

Randles PW, Libersky LD (1996) Smoothed particle hydrodynamics: some recent improvements and applications. Comput Methods Appl Mech Eng 139:375-408

Randles PW, Libersky LD (2000) Normalized SPH with stress points. Int J Numer Methods Eng 48(10):1445-1462

Robinson M (2009) Turbulence and viscous mixing using smoothed particle hydrodynamics. Ph.D. thesis, University of Monash, Melbourne

Rognebakke OF, Hoff JR, Allers JM, Berget K, Bergo BO, Zhao R (2006) Experimental approaches for determining sloshing loads in LNG tanks. Trans Soc Naval Archit Mar Eng 113:384-401

Rong S, Chen B (2010) Numerical simulation of Taylor bubble formation in micro-channel by MPS method. Microgravity Sci Technol 22:321-327

Saitoh TR, Makino J (2013) A density independent formulation of smoothed particle hydrodynamics. Am Astron Soc Astrophys J 768:24. doi:10.1088/0004-637X/768/1/44

Schwaiger HF (2008) An implicit corrected SPH formulation for thermal diffusion with linear free surface boundary conditions. Int J Numer Methods Eng 75(6):647-671

Scolan YM (2004) Hydroelastic behavior of a conical shell impacting on a quiescent-free surface of an incompressible liquid. J Sound Vib 277:163-203

Shadloo MS, Zainali A, Yildiz M, Suleman A (2012) A robust weakly compressible SPH method and its comparison with an incompressible SPH. Int J Numer Methods Eng 89(8):939-956

Shadloo MS, Weiss R, Yildiz M, Dalrymple RA (2015) Numerical simulation of long wave runup for breaking and nonbreaking waves. Int J Offshore Polar Eng 25(1):1-7

Shahriari S, Hassan IG, Kadem L (2013) Modeling unsteady flow characteristics using smoothed particle hydrodynamics. Appl Math Model 37(3):1431-1450

Shakibaeinia A, Jin YC (2012) MPS mesh-free particle method for multiphase flows. Comput Methods Appl Mech Eng 229:13-26

Shao S, Ji C, Graham DI, Reeve DE, James PW, Chadwick AJ (2006) Simulation of wave overtopping by an incompressible SPH model. Coast Eng 53(9):723-735

Shao SD (2010) Incompressible SPH flow model for wave interactions with porous media. Coast Eng 57:304-316

Shao SD (2012) Incompressible smoothed particle hydrodynamics simulation of multifluid flows. Int J Numer Methods Fluid 69:17151735

Shao S, Gotoh H (2004) Simulating coupled motion of progressive wave and floating curtain wall by SPH-LES model. Coast Eng J 46(2):171-202

Shao S, Lo EYM (2003) Incompressible SPH method for simulating Newtonian and non-Newtonian flows with a free surface. Adv Water Resour 26:787-800
Shao Y, Yamakwa T, Kikuchi T, Shibata K, Koshizuka S (2013) A three-dimensional coupling method for fluid-structure interaction problems by using explicit MPS method and Hamiltonian MPS method. Trans JSCES 20130004 (in Japanese). doi:10.11421/ jsces.2013.20130004

Shibata K, Koshizuka S, Sakai M, Tanizawa K (2011) Transparent boundary condition for simulating nonlinear water waves by a particle method. Ocean Eng 38(16):1839-1848

Shibata K, Koshizuka S, Sakai M, Tanizawa K (2012) Lagrangian simulations of ship-wave interactions in rough seas. Ocean Eng 42:13-25

Shibata K, Koshizuka S (2007) Numerical analysis of shipping water impact on a deck using a particle method. Ocean Eng 34(3):585593

Shirakawa S, Horie H, Yamamoto Y (1999) A study on the evaluation method of flow regimes with the particle interaction method. In: 7th International conference on nuclear engineering, pp 19-23

Skillen A, Lind S, Stansby PK, Rogers BD (2013) Incompressible smoothed particle hydrodynamics (SPH) with reduced temporal noise and generalised Fickian smoothing applied to body-water slam and efficient wave-body interaction. Comput Methods Appl Mech Eng 265:163-173

Souto-Iglesias A, Macià F, González LM, Cercos-Pita JL (2013) On the consistency of MPS. Comput Phys Commun 184(3):732-745

Sueyoshi M, Kashiwagi M, Naito S (2008) Numerical simulation of wave-induced nonlinear motions of a two-dimensional floating body by the moving particle semi-implicit method. J Mar Sci Technol 13(2):85-94

Sun JW, Liang SX, Sun ZC, Zhao XZ (2010) Simulation of wave impact on a horizontal deck based on SPH method. J Mar Sci Appl 9(4):372-378

Suzuki Y, Koshizuka S, Oka Y (2007) Hamiltonian moving-particle semi-implicit (HMPS) method for incompressible fluid flow. Comput Methods Appl Mech Eng 196:2876-2894

Swegle JW, Attaway SW, Heinstein MW, Mello FJ, Hicks DL (1994) An analysis of smooth particle hydrodynamics. Sandia Report SAND93-2513

Swegle JW (2000) Conservation of momentum and tensile instability in particle methods. Sandia Report SAND2000-1223

Swegle JW, Hicks DL, Attaway SW (1995) Smoothed particle hydrodynamics stability analysis. J Comput Phys 116:123-134

Szymczak W (1994) Energy losses in non-classical free surface flows. In: Blake J, Boulton-Stone J, Thomas N (eds) Bubble dynamics and interface phenomena, Fluid mechanics and its applications, vol 23. Springer, Netherlands, pp 413-420

Tartakovsky A, Meakin P (2005) Modeling of surface tension and contact angles with smoothed particle hydrodynamics. Phys Rev E 72(2):026301

Tartakovsky A, Panchenko A (2016) Pairwise force smoothed particle hydrodynamics model for multiphase flow: surface tension and contact line dynamics. J Comput Phys 305:1119-1146

Tayebi A, Jin YC (2015) Development of moving particle explicit (MPE) method for incompressible flows. Comput Fluids 117:1-10

Tiwari S, Klar A, Hardt S (2016) Numerical simulation of wetting phenomena by a meshfree particle method. J Comput Appl Math 292:469-485

Tofighi N, Ozbulut M, Rahmat A, Feng JJ, Yildiz M (2015) An incompressible smoothed particle hydrodynamics method for the motion of rigid bodies in fluids. J Comput Phys 297:207-220

Toyoshi T, Wada Y, Kikuchi M (2011) Solid-liquid flows simulation for debris avalanche analysis. Key Eng Mater 462 \& 463:855-860

Tsuruta N, Khayyer A, Gotoh H (2013) A short note on dynamic stabilization of moving particle semi-implicit method. Comput Fluids 82:158-164 
Tsuruta N, Khayyer A, Gotoh H (2015) Space potential particles to enhance the stability of projection-based particle methods. Int $\mathbf{J}$ Comput Fluid Dyn 29(1):100-119

Ulrich C, Leonardi M, Rung T (2013) Multi-physics SPH simulation of complex marine-engineering hydrodynamic problems. Ocean Eng 64:109-121

Vacondio R, Rogers BD, Stansby PK, Mignosa P (2016) Variable resolution for SPH in three dimensions: towards optimal splitting and coalescing for dynamic adaptivity. Comput Methods Appl Mech Eng 300:442-460

Verhagen JHG (1967) The impact of a flat plate on a water surface. J Ship Res 11(4):211-223

Vignjevic R (2004) Review of development of the smooth particle hydrodynamics (SPH) method. In: Proceedings of the 6th Conference on dynamics and control of systems and structures in space, Rimaggiore

Vignjevic R, Reveles JR, Campbell J (2006) SPH in a total Lagrangian formalism. Comput Model Eng Sci 4(3):181

Violeau D (2012) Fluid mechanics and the SPH method, theory and applications. Oxford University Press. ISBN: 978-0-19-965552-6

Violeau D, Buvat C, Abed-Meraim K, de Nanteuil E (2007) Numerical modelling of boom and oil spill with SPH. Coast Eng 54(12):895913

Violeau D, Issa R (2007) Numerical modelling of complex turbulent free-surface flows with the SPH method: an overview. Int J Numer Methods Fluids 53:277-304

Violeau D, Leroy A (2014) On the maximum time step in weakly compressible SPH. J Comput Phys 256:388-415

Violeau D, Leroy A (2015) Optimal time step for incompressible SPH. J Comput Phys 288:119-130

Violeau D, Rogers BD (2016) Smoothed particle hydrodynamics (SPH) for free-surface flows: past, present and future. J Hydraul Res 54(1):1-26

Wei Z, Dalrymple RA, Hérault A, Bilotta G, Rustico E, Yeh H (2015) SPH modeling of dynamic impact of tsunami bore on bridge piers. Coast Eng 104:26-42
Wendland H (1995) Piecewise polynomial, positive definite and compactly supported radial functions of minimal degree. Adv Comput Math 4:389-396

Xenakis AM, Lind SJ, Stansby PK, Rogers BD (2015) An incompressible SPH scheme with improved pressure predictions for free-surface generalised Newtonian flows. J Non Newton Fluid Mech 218:1-15

Xie J, Nistor L, Murty T (2012) A corrected 3-D SPH method for breaking tsunami wave modelling. Nat Hazards 60(1):81-100

Xu F, Chen JS, Huang QQ (2008) The study of numerical stability in the SPH method. Adv Mater Res 33-37:839-844

Xu R, Stansby PK, Laurence D (2009) Accuracy and stability in incompressible SPH (ISPH) based on the projection method and a new approach. J Comput Phys 228(18):6703-6725

Yang Q, Jones V, McCue L (2012) Free-surface flow interactions with deformable structures using an SPH-FEM model. Ocean Eng 55:136-147

Zanganeh M, Yeganeh-Bakhtiary A, Wahab AKA (2012) Lagrangian coupling two-phase flow model to simulate current-induced scour beneath marine pipelines. Appl Ocean Res 38:64-73

Zhang S, Morita K, Fukuda K, Shirakawa N (2007) A new algorithm for surface tension model in moving particle methods. Int J Numer Methods Fluids 55:225-240

Zhang S, Guo LC, Morita K, Fukuda K, Shirakawa N, Yamamoto Y (2008) Simulation of single bubble rising up in stagnant liquid pool with finite volume particle method. In: Sixth Japan-Korea symposium on nuclear thermal hydraulics and safety, pp 24-27

Zheng X, Ma QW, Duan WY (2014a) Comparative study of different SPH schemes on simulating violent water wave impact flows. China Ocean Eng 6(28):791-806

Zheng X, Ma QW, Duan WY (2014b) Incompressible SPH method based on Rankine source solution for violent water wave simulation. J Comput Phys 276:291-314 